\title{
ON MULTILINEAR OSCILLATORY INTEGRALS, NONSINGULAR AND SINGULAR
}

\author{
MICHAEL CHRIST, XIAOCHUN LI, TERENCE TAO, AND CHRISTOPH THIELE
}

\begin{abstract}
Basic questions concerning nonsingular multilinear operators with oscillatory factors are posed and partially answered. $L^{p}$ norm inequalities are established for multilinear integral operators of Calderón-Zygmund type which incorporate oscillatory factors $e^{i P}$, where $P$ is a real-valued polynomial.
\end{abstract}

\section{INTRODUCTION}

Consider multilinear functionals

$$
\Lambda_{\lambda}\left(f_{1}, f_{2}, \cdots, f_{n}\right)=\int_{\mathbb{R}^{m}} e^{i \lambda P(x)} \prod_{j=1}^{n} f_{j}\left(\pi_{j}(x)\right) \eta(x) d x
$$

where $\lambda \in \mathbb{R}$ is a parameter, $P: \mathbb{R}^{m} \rightarrow \mathbb{R}$ is a measurable real-valued function, $m \geq 2$, and $\eta \in C_{0}^{1}\left(\mathbb{R}^{m}\right)$ is compactly supported. Each $\pi_{j}$ denotes the orthogonal projection from $\mathbb{R}^{m}$ to a linear subspace $V_{j} \subset \mathbb{R}^{m}$ of any dimension $\kappa<m$, and $f_{j}: V_{j} \rightarrow \mathbb{C}$ is always assumed to be locally integrable with respect to Lebesgue measure on $V_{j}$. We assume for simplicity that $\kappa$ is independent of $j$.

The integral $\Lambda_{\lambda}$ is well-defined if all $f_{j}$ belong to $L^{\infty}$, and satisfies $\left|\Lambda_{\lambda}\left(f_{1}, \cdots, f_{n}\right)\right| \leq$ $C \prod_{j}\left\|f_{j}\right\|_{L^{\infty}}$.

Definition 1.1. A measurable real-valued function $P$ is said to have the power decay property, relative to a collection of subspaces $\left\{V_{j}\right\}$ of $\mathbb{R}^{m}$, on an open set $U \subset \mathbb{R}^{m}$ if for any $\eta \in C_{0}^{1}(U)$ there exist $\varepsilon>0$ and $C<\infty$ such that

$$
\left|\Lambda_{\lambda}\left(f_{1}, \cdots, f_{n}\right)\right| \leq C(1+|\lambda|)^{-\epsilon} \prod_{j=1}^{n}\left\|f_{j}\right\|_{L^{\infty}} \text { for all } f_{j} \in L^{\infty}\left(V_{j}\right) \text { and all } \lambda \in \mathbb{R} \text {. }
$$

Moreover, for $\eta$ supported in any fixed compact set, $\varepsilon$ may be taken to be independent of $\eta$, and $C=O\left(\|\eta\|_{C^{1}}\right)$.

Our goal is to characterize those data $\left(P,\left\{V_{j}\right\}\right)$ for which the power decay property holds. This problem has been well studied in the bilinear case where $n=2$, and to a lesser but still appreciable extent when $\kappa=1$ and $n=m$, but in the general case basic issues seem not to have been thoroughly explored.

A necessary condition for power decay is that $P$ can not be expressed as a linear combination of measurable functions $p_{j} \circ \pi_{j}$. Indeed, if $P=\sum_{j} p_{j} \circ \pi_{j}$ then for $f_{j}=e^{-i \lambda p_{j}} \in L^{\infty}$ one has $e^{i \lambda P(x)} \prod_{j} f_{j}\left(\pi_{j}(x)\right) \equiv 1$, and there is consequently no decay.

Date: November 16, 2004.

The first author was supported in part by NSF grants DMS-9970660 and 040126.

The second author was supported by NSF grants DMS-0140376 and DMS-011129.

The third author is a Clay Prize Fellow and is supported by a grant from the Packard foundation.

The fourth author was supported by NSF grants DMS 9970469 and DMS 9985572. 
Definition 1.2. A polynomial $P$ is said to be degenerate (relative to $\left\{V_{j}\right\}$ ) if there exist polynomials $p_{j}: V_{j} \rightarrow \mathbb{R}$ such that $P=\sum_{j=1}^{n} p_{j} \circ \pi_{j}$. Otherwise $P$ is nondegenerate.

In the case $n=0$, where the collection of subspaces $\left\{V_{j}\right\}$ is empty, $P$ is considered to be nondegenerate if and only if it is nonconstant.

We will show in Lemma 3.4 that degeneracy is also equivalent to the existence, in some nonempty open set, of a representation of the more general form $P=\sum_{j=1}^{n} h_{j} \circ \pi_{j}$ where the $h_{j}$ are arbitrary distributions rather than polynomials.

More generally, a (measurable) function $P$ is said to be degenerate if it can be expressed as $\sum_{j} p_{j} \circ \pi_{j}$ for some (measurable) functions $p_{j}$, or distributions. But we will restrict attention to polynomials henceforth.

Question. Is the power decay property equivalent to nondegeneracy, for real-valued polynomials?

We were led to this question by a problem concerning multilinear singular integral operators; see Theorems 2.5 and 2.6 below. We have been able to answer it, always in the affirmative, only in special cases.

Numerous variants can be formulated. One can ask when (1.2) holds with the right-hand side replaced by $\Theta(\lambda) \prod_{j=1}^{n}\left\|f_{j}\right\|_{L^{\infty}}$ where $\Theta(\lambda)$ is a specific function of $(1+|\lambda|)$, or whether there merely exists some function $\Theta$ tending to zero as $|\lambda| \rightarrow \infty$ for which it holds. When (1.2) does hold, one can ask what is the optimal power $\varepsilon$ in (1.2). We will focus on the first formulation, which is the one most directly relevant to our applications to multilinear singular integral operators, and is possibly the most fundamental.

The extreme formulation in which all $f_{j}$ are measured in the strongest Lebesgue norm $L^{\infty}$ is the essence of the matter. Since $\left|\Lambda_{\lambda}\left(f_{1}, \cdots, f_{n}\right)\right| \leq C\left\|f_{k}\right\|_{L^{1}} \prod_{j \neq k}\left\|f_{j}\right\|_{L^{\infty}}$ uniformly in $\lambda$ for any $k$, a simple interpolation argument shows that if (1.2) does hold, then a decay estimate of the same type holds with $\prod_{j}\left\|f_{j}\right\|_{\infty}$ replaced by $\prod_{j}\left\|f_{j}\right\|_{p_{j}}$ for various $n$-tuples of indices $p_{j} \in(1, \infty]$. More precisely, if the integral converges absolutely whenever each $f_{j} \in L^{p_{j}}$, then $\left|\Lambda_{\lambda}\left(f_{1}, \cdots, f_{n}\right)\right| \leq C(1+|\lambda|)^{-\varepsilon} \prod_{j}\|f\|_{q_{j}}$ whenever each $q_{j}>p_{j}$, where $\varepsilon>0$ depends on $\left\{p_{j}\right\},\left\{q_{j}\right\}$, and other $n$-tuples $q$ can in general be obtained via further interpolations. On the other hand, the combinations of exponents $p_{j}$ for which the integral is guaranteed to converge absolutely depend strongly on $\left\{V_{j}\right\}$.

When $n=0$, that is, when $\left\{V_{j}\right\}$ is empty, one is dealing with oscillatory integrals $\Lambda_{\lambda}=$ $\int e^{i \lambda P(x)} \eta(x) d x$, which are of the first type in the terminology of Stein [10]. The general case can also be regarded as concerning oscillatory integrals of the first type. Indeed, (1.2) with $L^{\infty}$ bounds on the functions $f_{j}$ is equivalent to $\left|\int e^{i \lambda \phi(x)} \eta(x) d x\right| \leq C|\lambda|^{-\epsilon}$ uniformly for all phase functions of the form $\phi=P-\sum_{j} h_{j} \circ \pi_{j}$, where the $h_{j}$ are arbitrary real-valued measurable functions; one implication is tautologous, while the converse is nearly trivial and is proved in [1].

The regularity condition $\eta \in C^{1}$ is rather arbitrary. If $\eta$ is merely Hölder continuous then for any $s<\infty, \eta$ may be decomposed as a smooth function whose $C^{s}$ norm is $O\left(|\lambda|^{C \delta}\right)$ plus a remainder which is $O\left(|\lambda|^{-\delta}\right)$ in supremum norm. If (1.2) holds for all $\eta \in C_{0}^{s}$ with a constant $C$ which is $O\left(\|\eta\|_{C^{s}}\right)$ then it follows from this decomposition, with $\delta=\varepsilon / 2 C$, that it continues to hold for all Hölder continuous $\eta$.

$\prod_{j=1}^{n} f_{j} \circ \pi_{j}$ could be replaced by $\prod_{j=1}^{n} g_{j} \circ \ell_{j}$ in (1.1), where each $\ell: \mathbb{R}^{m} \rightarrow \mathbb{R}^{\kappa}$ is linear and has full rank, without any increase in generality. For any function $g \circ \ell$ may be expressed as $f \circ \pi$, where $\pi$ is the orthogonal projection of $\mathbb{R}^{m}$ onto the orthocomplement $V$ of the nullspace of $\ell ; f \in L^{p}(V)$ if and only if $g \in L^{p}\left(\mathbb{R}^{\kappa}\right)$ with comparable norms. 
The linear prototype for power decay is the inequality (see Stein [10], p. 416 and Phong and Stein [7])

$$
\left|\int e^{i \lambda P(x, y)} f(x) g(y) \eta(x, y) d x d y\right|=O\left(|\lambda|^{-\varepsilon}\|f\|_{L^{2}}\|g\|_{L^{2}}\right),
$$

with suitable uniformity, whenever $P$ is a real-valued polynomial, of bounded degree, which is nondegenerate in the sense that some mixed partial derivative $\partial^{\alpha+\beta} P / \partial x^{\alpha} \partial y^{\beta}$, with both $\alpha, \beta \neq 0$, does not vanish identically; this is equivalent to the impossibility of decomposing $P(x, y)$ as $p(x)+q(y)$.

A related result is the lemma of van der Corput, which asserts for instance that $\int_{a}^{b} e^{i \lambda \varphi(t)} d t=$ $O(1+|\lambda|)^{-\varepsilon}$ provided that $\varphi$ is real-valued and that some derivative of $\varphi$ is bounded away from zero (in the case of the first derivative, it is also assumed to be monotone). Here the upper bound for the integral depends only on a lower bound for the derivative; no upper bound on $\varphi$ or its derivatives, hence in some sense no a priori smoothness condition, are imposed. Higher-dimensional versions of van der Corput's lemma, likewise without assumptions of upper bounds or extra smoothness, were established in [1].

Our nondegeneracy condition replaces the hypothesis of a nonvanishing derivative. (1.2), like (1.3), is invariant under the replacement of $P$ by $P-\sum_{j} p_{j} \circ \pi_{j}$, for arbitrary real-valued measurable functions $p_{j}$, and there is no a priori smoothness condition or upper bound on $P-\sum_{j} p_{j} \circ \pi_{j}$. Formally the power decay property more closely resembles (1.3), but our analysis is closely related to [1], and combinatorial issues lurk here as they did there.

\section{Results}

2.1. Further definitions. Given a degree $d$, fix a norm $\|\cdot\|_{\mathcal{P}_{d}}$ on the finite-dimensional vector space $\mathcal{P}_{d}$ of all polynomials in $\mathbb{R}^{m}$ of degree $\leq d$. Given $d$, the norm of $P$ relative to a finite collection of linear subspaces $\left\{V_{j}\right\}$ is defined to be inf $\left\|P-\sum_{j} p_{j} \circ \pi_{j}\right\|_{\mathcal{P}_{d}}$, where the infimum is taken over all real-valued polynomials $p_{j}$ of degrees $\leq d$. Polynomials $P_{\alpha}$ are said to be uniformly nondegenerate if they are all of degrees $\leq d$ for some finite $d$, and if there exists $c>0$ such that the norm of $P_{\alpha}$ relative to $\left\{V_{j}\right\}$ (and relative to this degree $d)$ is $\geq c$ for all $\alpha$.

More generally, if we are given given a finite collection of subspaces $\left\{V_{j}^{\alpha}: \alpha \in A\right\}$ and a polynomial $P_{\alpha}$ of uniformly bounded degree, we may still define uniform nondegeneracy by requiring that $\inf _{\alpha} \inf _{\left\{p_{j}\right\}}\left\|P_{\alpha}-\sum_{j} p_{j} \circ \pi_{j}^{\alpha}\right\|_{\mathcal{P}_{d}} \geq c>0$, where $\pi_{j}^{\alpha}$ is the orthogonal projection onto $V_{j}^{\alpha}$.

These definitions are independent of $d$ provided only that each $P$ has degree $\leq d$, a simple consequence of the equivalence of any two norms on a finite-dimensional vector space, and the fact that if $P=\sum_{j} p_{j} \circ \pi_{j}$ then $P=\sum_{j} \tilde{p}_{j} \circ \pi_{j}$ where $\tilde{p}_{j}$ is the sum of all terms of degrees $\leq$ degree $(P)$ in the decomposition of $\pi_{j}$ as a linear combination of monomials.

Because the family of polynomials of degree $\leq d$ has finite dimension, it is easily verified that the infimum defining the relative norm is actually assumed for some polynomials $p_{j}$. Thus either $P$ is degenerate, or the infimum is strictly positive.

Definition 2.1. A finite collection of subspaces $\left\{V_{j}\right\}$ is said to have the power decay property if every real-valued polynomial $P$ which is nondegenerate relative to $\left\{V_{j}\right\}$ has the power decay property (1.2), in every open set $U$.

A collection of subspaces $\left\{V_{j}\right\}$ is said to have the uniform power decay property if (1.2) holds, with uniform constants $C, \varepsilon$, for any family of real-valued polynomials of bounded degrees which are uniformly nondegenerate relative to $\left\{V_{j}\right\}$. 
A related concept turns out to be somewhat easier to analyze. To any distribution $h \in \mathcal{D}^{\prime}\left(V_{j}\right)$ is naturally associated a distribution $h \circ \pi_{j} \in \mathcal{D}^{\prime}\left(\mathbb{R}^{m}\right)$. Denote by $V_{j}^{\perp}$ the orthogonal complement of $V_{j}$. Then $h \circ \pi_{j}$ is annihilated by any first-order constantcoefficient differential operators of the form $w \cdot \nabla$, where $w \in V_{j}^{\perp}$.

Definition 2.2. A polynomial $P$ is said to be simply nondegenerate relative to $\left\{V_{j}: 1 \leq\right.$ $j \leq n\}$ if there exists a differential operator $L$ of the form $L=\prod_{j=1}^{n}\left(w_{j} \circ \nabla\right)$, with each $w_{j} \in V_{j}^{\perp}$, such that $L(P)$ does not vanish identically.

Simple nondegeneracy implies nondegeneracy. Indeed, for any distribution $f$ defined on some $V_{j}$, the $j$-th factor of $L$ annihilates $f \circ \pi_{j}$, and hence $L$ does so since all factors commute. Thus $L\left(P-\sum_{j} f_{j} \circ \pi_{j}\right) \equiv L(P)$.

The converse is not true in general. Consider the case $\kappa=1$ where each subspace $V_{j}$ has dimension one. Let $\mathcal{L}$ be any homogeneous constant-coefficient linear partial differential operator with real coefficients, and let $\sigma$ be its symbol. Identify the dual space of $\mathbb{R}^{m}$ with $\mathbb{R}^{m}$ via the inner product. Let $e$ be any unit vector and let $\pi(x)=\langle x, e\rangle e$. Then $\mathcal{L}$ annihilates every function $f \circ \pi$ if and only if $\sigma(e)=0$. To create examples of nondegenerate polynomials, fix any such nonelliptic $\mathcal{L}$, choose $\left\{e_{j}\right\}$ to be any finite collection of distinct unit vectors satisfying $\sigma\left(e_{j}\right)=0$, and choose any real-valued polynomial $P$ such that $\mathcal{L}(P)$ does not vanish identically. This forces $P$ to be nondegenerate relative to $\left\{V_{j}\right\}$, where $V_{j}$ is the span of $e_{j}$. If $m \geq 3$ then $P, \mathcal{L},\left\{V_{j}\right\}$ can be chosen so that $P$ has degree two, yet $\left\{V_{j}\right\}$ has arbitrarily large finite cardinality.

2.2. Decay for nonsingular oscillatory multilinear functionals. We now state several theorems asserting that nondegeneracy implies the power decay property, under various auxiliary hypotheses. To formulate the first of these, let $\left\{V_{j}: 1 \leq j \leq n\right\}$ be a collection of subspaces of $\mathbb{R}^{m}$ of dimension $\kappa$. We say that they are in general position if any subcollection of cardinality $k \geq 1$ spans a subspace of dimension $\min (k \kappa, m)$. It is elementary that for subspaces in general position, if each $f_{j}$ belongs to $L^{2}(\mathbb{R})$, then the product of any $k$ functions $f_{j} \circ \pi_{j}$ belongs to $L^{2}\left(\mathbb{R}^{m}\right)$ provided that $k \kappa \leq m$. When $k \kappa \leq 2 m$ the product belongs to $L^{1}$, by Cauchy-Schwarz. Therefore the integral defining $\Lambda_{\lambda}$ converges absolutely, and $\left|\Lambda_{\lambda}\left(f_{1}, \cdots, f_{n}\right)\right| \leq C \prod_{j}\left\|f_{j}\right\|_{L^{2}}$, uniformly in $\lambda \in \mathbb{R}$.

Theorem 2.1. Suppose that $n<2 m$. Then any family $\left\{V_{j}: 1 \leq j \leq n\right\}$ of one-dimensional subspaces of $\mathbb{R}^{m}$ which lie in general position has the uniform power decay property. Moreover under these hypotheses

$$
\left|\Lambda_{\lambda}\left(f_{1}, \cdots, f_{n}\right)\right| \leq C(1+|\lambda|)^{-\varepsilon} \prod_{j=1}^{n}\left\|f_{j}\right\|_{L^{2}}
$$

for all polynomials $P$ of bounded degree which are uniformly nondegenerate with respect to $\left\{V_{j}\right\}$, for all functions $f_{j} \in L^{2}\left(\mathbb{R}^{1}\right)$, with uniform constants $C, \varepsilon \in \mathbb{R}^{+}$.

The case $n=m$ is known; Phong, Stein, and Sturm [8] have obtained much more precise results on the exponent in the power decay estimate, phrased in terms of the reduced Newton polyhedron of $P$. The case $n<m$ follows from $n=m$. Indeed, choose coordinates $\left(x^{\prime}, x^{\prime \prime}\right) \in \mathbb{R}^{n} \times \mathbb{R}^{m-n}$, in such a way that the first factor $\mathbb{R}^{n}$ equals the span of the subspaces $V_{j}$. If $\partial P / \partial x^{\prime \prime}$ does not vanish identically then (1.2) does hold, as one sees by integrating with respect to $x^{\prime \prime}$ for generic fixed $x^{\prime}$ and using well-known results for oscillatory integrals of the first type in the sense of [10]. If $P$ is independent of $x^{\prime \prime}$ then matters have been reduced to the case where the ambient dimension is $n$. 
As will be clear from its proof, (2.1) continues to hold with uniform constants $C, \varepsilon$ if the collection $\left\{V_{j}\right\}$ varies over a compact subset of the open subset of the relevant Grassmannian manifold consisting of all such collections in general position, and the polynomials $P$ are uniformly nondegenerate relative to $\left\{V_{j}\right\}$.

The condition $n<2 m$ is necessary for (2.1), with $\left\|f_{j}\right\|_{L^{2}}$ on the right-hand side rather than $\left\|f_{j}\right\|_{L^{\infty}}$ as in Definition 1.1. To see this in the main case where $\left\{V_{j}\right\}$ span $\mathbb{R}^{m}$, consider without loss of generality the case $n=2 m$, and let each $f_{j}$ equal the characteristic function of $[-\delta, \delta]$. If $\delta$ is chosen to be a small fixed constant times $|\lambda|^{-1}$, then $\left|\Lambda_{\lambda}\left(f_{1}, \cdots, f_{n}\right)\right| \geq c \delta^{m}$. On the other hand $\prod_{j}\left\|f_{j}\right\|_{L^{2}}=c^{\prime} \delta^{n / 2}$. It follows from the same construction that if $n>2 m$ then the integral defining $\Lambda_{\lambda}\left(f_{1}, \cdots, f_{n}\right)$ will in general not even be absolutely convergent for general $f_{j} \in L^{2}$.

Theorem 2.2. Any collection of subspaces $\left\{V_{j}\right\}$ of codimension one has the uniform power decay property.

In particular, when $m=2$ then $\kappa=1=m-1$ and hence nondegeneracy of $P$ implies (1.2), no matter how large the collection of subspaces $V_{j}$ may be.

Theorem 2.3. Let $m, \kappa$ be arbitrary. Then any simply nondegenerate polynomial has the power decay property (1.2) in every open set.

More precisely, let $d \in \mathbb{N}$ and $c>0$. Let $L=\prod_{j=1}^{n}\left(w_{j} \cdot \nabla\right)$ where each $w_{j} \in V_{j}^{\perp}$ is a unit vector. Then there exist $C<\infty$ and $\varepsilon>0$ such that for any real-valued polynomial $P$ of degree $\leq d$ such that $\max _{|x| \leq 1}|L(P)(x)| \geq c$,

$$
\left|\Lambda_{\lambda}\left(f_{1}, \cdots, f_{n}\right)\right| \leq C(1+|\lambda|)^{-\varepsilon} \prod_{j}\left\|f_{j}\right\|_{\infty}
$$

for all functions $f_{j} \in L^{\infty}$ and all $\lambda \in \mathbb{R}$.

In each of these theorems, the regularity hypothesis on $\eta$ can be relaxed to Hölder continuity, as discussed above.

Theorem 2.2 directly implies Theorem 2.3. Indeed, if $w_{j}$ are as in the latter theorem, define $\tilde{V}_{j}=w_{j}^{\perp}$, thus obtaining codimension one subspaces $\tilde{V}_{j} \supset V_{j}$. Each $f_{j} \circ \pi_{j}$ can be rewritten as $\tilde{f}_{j} \circ \tilde{\pi}_{j}$ where $\tilde{\pi}_{j}$ is the orthogonal projection onto $\tilde{V}_{j}$ and $\tilde{f}_{j}(y)=f_{j} \circ \pi_{j}(y)$ for $y \in \tilde{V}_{j}$. The hypothesis $L(P) \neq 0$ guarantees that $P$ is nondegenerate relative to $\left\{\tilde{V}_{j}\right\}$, with appropriate uniformity. On the other hand, we will show in Proposition 3.1 that for $\kappa=m-1$, nondegeneracy is equivalent to simple nondegeneracy, so that Theorem 2.2 conversely implies Theorem 2.3.

The next result concerns the case of small codimension, but is less satisfactory than the definitive statement of Theorem 2.2 for codimension one.

Theorem 2.4. Let $\left\{V_{j}: 1 \leq j \leq n\right\}$ be a finite collection of subspaces of $\mathbb{R}^{m}$, each having dimension $\kappa$ and lying in general position. If

$$
n \leq \frac{m}{m-\kappa}
$$

then $\left\{V_{j}\right\}$ has the uniform decay property.

In this result, general position of $\left\{V_{j}\right\}$ means instead that the orthocomplements $\left\{V_{j}^{\perp}\right\}$ lie in general position in the sense defined above, that is, their joint span has dimension $\leq$ $(m-\kappa) n$. Lemma 3.5 will assert that nondegeneracy is equivalent to simple nondegeneracy when $n \leq \frac{m}{m-\kappa}$, so that Theorem 2.4 will be a corollary of Theorem 2.3. 
2.3. Singular integrals. For any real valued polynomial $P(x, t)$ of degree $d$, consider the operator

$$
T(f, g)(x)=\int_{-\infty}^{\infty} e^{i P(x, t)} f(x-t) g(x+t) t^{-1} d t
$$

where it is initially assumed that $f, g \in C_{0}^{1}$, the class of all continuously differentiable functions having compact supports, and the integral is taken in the principal-value sense. One of the purposes of this note is to establish the following $L^{p}$ bounds for these operators.

Theorem 2.5. For any exponents $p_{1}, p_{2}, q \in(0, \infty)$ such that $q^{-1}=p_{1}^{-1}+p_{2}^{-1}, p_{1}, p_{2}>1$ and $q>2 / 3$, and any degree $d \geq 1$, there exists $C<\infty$ such that $\|T(f, g)\|_{q} \leq C\|f\|_{p_{1}}\|g\|_{p_{2}}$ for all $f, g \in C_{0}^{1}$, uniformly for all real-valued polynomials $P$ of degrees $\leq d$.

This answers a question raised by Lacey and Thiele. The cases $d=0,1,2$ were previously known. Indeed, the case $d=0$ is a celebrated theorem of Lacey and Thiele [4]. The case $d=1$, that is $P(x, t)=a_{0} x+a_{1} t$, can be reduced to $d=0$ by replacing $f$ by $\tilde{f}(x)=e^{i a_{0} x / 2-i a_{1} x / 2} f(x)$ and $g$ by $\tilde{g}(x)=e^{i a_{0} x / 2+i a_{1} x / 2} f(x)$. The case $d=2$, that is, $P(x, t)=a_{0} x^{2}+a_{1} x t+a_{2} t^{2}+b_{1} x+b_{2} t$, is likewise reducible to $d=0$ by the substitution $\tilde{f}(x)=e^{-i a_{1} x^{2} / 4+i a_{2} x^{2} / 2} f, \tilde{g}(x)=e^{i a_{1} x^{2} / 4+i a_{2} x^{2} / 2} g$. But for $d \geq 3$ no such simple reduction exists.

Theorem 2.5 is a bilinear analogue of a theorem of Ricci and Stein [9], who proved $L^{p}$ estimates for linear operators $f \mapsto \int e^{i P(x, t)} f(x-t) K(t) d t$, for arbitrary real-valued polynomials $P$ and Calderón-Zygmund kernels $K$. It is a special case of the following more general result. Let $n \geq 1, \Gamma=\left\{\xi \in \mathbb{R}^{n+1}: \xi_{1}+\xi_{2}+\cdots+\xi_{n+1}=0\right\}$, and $\Gamma^{\prime}$ be the orthogonal complement of a subspace of $\Gamma$ such that the dimension of $\Gamma^{\prime} \cap \Gamma$ is $k$. Let $K$ be a $k$-dimensional Calderón-Zygmund kernel on $\Gamma^{\prime} \cap \Gamma$, that is, $K$ is Lipschitz continuous except at the origin, $K(r \gamma) \equiv r^{-k} K(\gamma)$ for all $r>0$ and $\gamma \neq 0$, and $\int_{S^{k-1}} K d \sigma=0$ where $\sigma$ denotes surface measure on the unit sphere $S^{k-1}$ in $\Gamma^{\prime} \cap \Gamma$. We define the $n$-linear operator $T$ by

$$
T\left(f_{1}, f_{2}, \cdots, f_{n}\right)(x)=\int_{\Gamma^{\prime} \cap \Gamma} f_{1}\left(x+\gamma_{1}\right) f_{2}\left(x+\gamma_{2}\right) \cdots f_{n}\left(x+\gamma_{n}\right) K(\gamma) d \gamma
$$

for $x \in \mathbb{R}^{1}$, where $d \gamma$ is Lebesgue measure on $\Gamma^{\prime} \cap \Gamma$, and $\gamma_{i} \in \mathbb{R}^{1}$ is the $i$-th coordinate of $\gamma \in \mathbb{R} \times \mathbb{R} \cdots \times \mathbb{R}$ as an element of $\mathbb{R}^{n+1}$. The integral is interpreted in the principal-value sense, so that $T\left(f_{1}, \cdots, f_{n}\right)(x)$ is well-defined provided that each $f_{j} \in C^{1}$ has compact support. We always assume that any $k+1$ variables in $\left\{x+\gamma_{1}, \cdots, x+\gamma_{n}, x\right\}$ are linearly independent.

To such an operator $T$ and to any real-valued polynomial $P\left(x, \gamma_{1}, \cdots, \gamma_{n}\right)$ we associate the multilinear operator

$$
T_{P}\left(f_{1}, f_{2}, \cdots, f_{n}\right)(x)=\int_{\Gamma^{\prime} \cap \Gamma} e^{i P\left(x, \gamma_{1}, \cdots, \gamma_{n}\right)} f_{1}\left(x+\gamma_{1}\right) f_{2}\left(x+\gamma_{2}\right) \cdots f_{n}\left(x+\gamma_{n}\right) K(\gamma) d \gamma
$$

which is again well-defined when each $f_{j} \in C_{0}^{1}$.

Write $p_{0}=q^{\prime}$.

Theorem 2.6. Suppose that $n \leq 2 k$. Then for any real-valued polynomial $P, T_{P}$ maps $\otimes_{j=1}^{n} L^{p_{j}}$ boundedly to $L_{0}^{p}$ whenever $p_{0}>n^{-1}, 1<p_{j} \leq \infty$ and $p_{0}^{-1}=\sum_{j} p_{j}^{-1}$, provided that

$$
\frac{1}{p_{i_{1}}}+\frac{1}{p_{i_{2}}}+\cdots+\frac{1}{p_{i_{r}}}<\frac{2 k+r+1-n}{2}
$$


for all $0 \leq i_{1}<i_{2}<\cdots<i_{r} \leq n$ and all $1 \leq r \leq n+1$. This conclusion holds uniformly for all polynomials of degrees $\leq D$, for any $D<\infty$.

Under these hypotheses, the nonoscillatory case $P \equiv 0$ was treated in [6]. Theorems 2.5 and 2.6 will be proved by combining previously known results for nonoscillatory multilinear singular integral operators with our new results for nonsingular oscillatory integrals.

The remainder of the paper is devoted to proofs of the theorems. Certain algebraic aspects of nondegeneracy come into play in the analysis and are developed in $\S 3$; this material is used throughout the paper. The simply nondegenerate case is treated in $\S 4$. The analysis when the dimension $\kappa$ equals 1 involves an additional ingredient and is presented in $\S 5$. Applications to multilinear operators with Calderón-Zygmund singularities and oscillatory factors are carried out in $\S 6$. $\S 7$ is devoted to an extension of Theorem 2.2 and, as an application, a simpler reorganization of the proof of Theorem 2.1.

Our results are certainly not definitive. One known inequality not covered by our theorems is the twisted convolution inequality

$$
\left|\iint_{\mathbb{C}^{n+n}} f_{1}(z) f_{2}(z-w) f_{3}(w) e^{i \lambda \operatorname{Im}(z \cdot \bar{w})}\right| \leq C|\lambda|^{-n / 2} \prod_{j=1}^{3}\left\|f_{j}\right\|_{L^{2}\left(\mathbb{C}^{n}\right)},
$$

where the integration is with respect to Lebesgue measure on $\mathbb{C}^{n+n}$. See Proposition 3 , page 556 of [10].

Acknowledgement. The second author thanks M. Lacey for encouraging him to work on this project.

\section{Algebraic aspects of NOndegeneracy}

This section develops various general properties, largely algebraic in nature, of nondegeneracy. The first observation is that for given dimensions $m, \kappa$, degree of multilinearity $n$, and subspaces $V_{j}$, there exist nondegenerate homogeneous polynomials $P$ of all sufficiently high degrees. Indeed, the dimension of the vector space of all $\sum_{j} q_{j} \circ \pi_{j}$, where $q_{j}$ is an arbitrary homogeneous polynomial of degree $D$ on $\mathbb{R}^{\kappa}$, is $O\left(n D^{\kappa}\right)$, while the space of all homogeneous polynomials $P: \mathbb{R}^{m} \rightarrow \mathbb{R}$ is $\sim c D^{m} \gg D^{\kappa}$ as $D \rightarrow \infty$.

As noted above, simple nondegeneracy implies nondegeneracy. In the codimension one case they are equivalent:

Proposition 3.1. If each subspace $V_{j}$ has codimension one, that is if $\kappa=m-1$, then any nondegenerate polynomial is simply nondegenerate.

Proof. Let a polynomial $P$ and distinct subspaces $V_{j}$ of codimension 1 be given. Let $w_{j}$ be unit vectors orthogonal to $V_{j}$, let $L=\prod_{j=1}^{n}\left(w_{j} \cdot \nabla\right)$, and let $L^{\prime}=\prod_{j=1}^{n-1}\left(w_{j} \cdot \nabla\right)$. We must show that if $L P \equiv 0$, then $P$ is degenerate.

Proceed by induction on $n$, which is the total number of subspaces $V_{j}$. Then since $L^{\prime}\left(w_{n} \cdot \nabla\right) P \equiv 0,\left(w_{n} \cdot \nabla\right) P=\sum_{j=1}^{n-1} q_{j} \circ \pi_{j}$ for some polynomials $q_{j}$. By a rotation of the coordinate system it may be arranged that $w_{n} \cdot \nabla=\partial_{x_{m}}$.

$\left(w_{n} \cdot \nabla\right)\left(f \circ \pi_{j}\right)=\left(D_{j} f\right) \circ \pi_{j}$ for some nonvanishing constant-coefficient real vector fields $D_{j}$, for $w_{n}$ cannot be orthogonal to $V_{j}$ for $j<n$ since it is orthogonal to $V_{n}$, and these codimension one subspaces are distinct. Consequently there exist polynomials of the form $p_{j} \circ \pi_{j}$ for $1 \leq j \leq n-1$ such that $\left(w_{n} \cdot \nabla\right)\left(p_{j} \circ \pi_{j}\right)=q_{j} \circ \pi_{j}$. 
Thus $\left(w_{n} \cdot \nabla\right)\left(P-\sum_{j<n} p_{j} \circ \pi_{j}\right) \equiv 0$, so the polynomial $\left(P-\sum_{j<n} p_{j} \circ \pi_{j}\right)(x)$ is a function of $x_{m}=\pi_{m}(x)$ alone, and hence is of the form $p_{n} \circ \pi_{n}$. This proves that $P$ is degenerate.

Lemma 3.2. Let $P$ be a homogeneous polynomial of some degree $d$. Then $P$ is nondegenerate relative to a finite collection of subspaces $\left\{V_{j}\right\}$, of any dimension, if and only if there exists a constant-coefficient partial differential operator $\mathcal{L}$, homogeneous of degree $d$, such that $\mathcal{L}(P) \neq 0$ but $\mathcal{L}\left(p_{j} \circ \pi_{j}\right) \equiv 0$ for every polynomial $p_{j}: V_{j} \rightarrow \mathbb{C}$ of degree $d$.

Proof. If such an operator $\mathcal{L}$ exists then $P$ is obviously nondegenerate. To prove the converse, fix $d$ and denote by $\mathcal{P}_{d}$ the vector space of all homogeneous polynomials of degree $d$. The pairing $\langle\mathcal{L}, P\rangle=\mathcal{L}(P)$ between homogeneous constant-coefficient differential operators of the same degree $d$ and elements of $\mathcal{P}_{d}$ is nondegenerate. Thus the dual space of $\mathcal{P}_{d}$ may be canonically identified with the vector space of all such differential operators.

If $P$ is nondegenerate then it does not belong to the subspace of all degenerate homogeneous polynomials, so there exists a linear functional which annihilates that subspace, but not $P$. This functional may be realized as $Q \mapsto \mathcal{L}(Q)$ for some homogeneous constantcoefficient linear partial differential operator $\mathcal{L}$, completing the proof in the converse direction.

From this it is apparent that if $D$ and $\left\{\pi_{j}\right\}$ are held fixed, then the collection of all nondegenerate polynomials $P$ of degrees $\leq D$ is an open subset of the vector space of all polynomials of degrees $\leq D$. On the other hand, nondegeneracy is not an open condition on $\left\{\pi_{j}: 1 \leq j \leq n\right\}$; if $n$ is large relative to $m$ then the condition that there exist nontrivial differential operators annihilating all functions $q_{j} \circ \pi_{j}$ is unstable under small perturbations.

Any polynomial may be expressed in a unique way as a sum of homogeneous polynomials of distinct degrees.

Lemma 3.3. A polynomial $P$ is nondegenerate relative to $\left\{V_{j}\right\}$ if and only if at least one of its homogeneous summands is nondegenerate. Moreover, a homogeneous polynomial is degenerate if and only if it may be expressed as $\sum_{j} p_{j} \circ \pi_{j}$ where each $p_{j}$ is a homogeneous polynomial of the same degree.

Proof. Both of these assertions follow from the simple fact that a polynomial $p_{j}: \mathbb{R}^{\kappa} \rightarrow \mathbb{R}$ is homogeneous of some degree if and only if $p_{j} \circ \pi_{j}: \mathbb{R}^{k} \rightarrow \mathbb{R}$ is homogeneous, of the same degree.

If $P$ can be decomposed as $\sum_{j} f_{j} \circ \pi_{j}$ for some functions $h_{j}$ which are not necessarily polynomials, then the decay bound (1.2) certainly fails, for the same reason noted earlier (set $\left.f_{j}=e^{-i \lambda h_{j}}\right)$. The next lemma says that it makes no difference whether the $h_{j}$ are taken to be polynomials, or arbitrary functions, in the definition of nondegeneracy.

Lemma 3.4. A polynomial $P$ is degenerate with respect to a collection of subspaces $\left\{V_{j}\right\}$ if and only if there exist distributions $h_{j}$ in $\mathbb{R}^{\kappa}$ such that $P=\sum_{j} h_{j} \circ \pi_{j}$ in some open set.

Proof. Let $\left\{V_{j}\right\}$ be given, and let $P$ be any nondegenerate polynomial of some degree $d$. We need only show that $P$ can't be decomposed locally as $\sum_{j} h_{j} \circ \pi_{j}$, since the converse is a tautology. It is no loss of generality to suppose that the homogeneous summand of $P$ of degree $d$ is nondegenerate. For otherwise we may express that summand as $\sum_{j} p_{j} \circ \pi_{j}$ where the $p_{j}$ are homogeneous polynomials of degree $d$, then replace $P$ by $P-\sum_{j} p_{j} \circ \pi_{j}$ to reduce the degree. 
By Lemma 3.2, there exists a constant-coefficient linear partial differential operator $\mathcal{L}$, homogeneous of degree $d$, such that $\mathcal{L}(P) \neq 0$, yet $\mathcal{L}\left(p_{j} \circ \pi_{j}\right)=0$ for any polynomial $p_{j}$ homogeneous of degree $d$, for any index $j$.

$\mathcal{L}(Q)(0)=0$ for any polynomial $Q: \mathbb{R}^{m} \rightarrow \mathbb{R}$ which is homogeneous of any degree except $d$. By combining this with the hypothesis we find that $\mathcal{L}\left(p \circ \pi_{j}\right)(0)=0$ for any polynomial $p: \mathbb{R}^{\kappa} \rightarrow \mathbb{R}$ and any index $j$. Consequently $\mathcal{L}\left(p \circ \pi_{j}\right)(x)=0$ for all $x \in \mathbb{R}^{m}$. Since any distribution is a limit of polynomials in the distribution topology, $\mathcal{L}\left(h_{j} \circ \pi_{j}\right)$ likewise vanishes identically, in the sense of distributions. Yet $\mathcal{L}$ does not annihilate $P$, so $P$ cannot be decomposed as $\sum_{j} h_{j} \circ \pi_{j}$.

In Lemmas 3.5 and 3.6 we say that a polynomial is a monomial if it can be expressed as a product of linear factors. Thus $\left(x_{1}+x_{2}\right) x_{1}^{2} x_{3}$ is a monomial. By a differential monomial we mean a linear partial differential operator which can be expressed as a product of finitely many real vector fields, each with constant coefficients.

Lemma 3.5. Let $\left\{V_{j}: 1 \leq j \leq n\right\}$ be a finite collection of subspaces of $\mathbb{R}^{m}$, each having dimension $\kappa$ and lying in general position. If $(m-\kappa) n \leq m$ then any polynomial which is nondegenerate relative to $\left\{V_{j}\right\}$ is simply nondegenerate relative to $\left\{V_{j}\right\}$.

In Lemmas 3.5 and 3.6, general position means that the orthocomplements $V_{j}^{\perp}$ are linearly independent, that is, together span a space of dimension $(m-\kappa) n$. This only makes sense under the hypothesis that $(m-\kappa) n \leq m$.

Proof. Consider the variety $\mathcal{V}=\cup_{j} V_{j}$. Let $\mathcal{I}$ be the ideal in $\mathbb{C}[x]$ consisting of all real polynomials which vanish on $\mathcal{V}$. Then $\mathcal{I}$ is of course finite-dimensional as a $\mathbb{C}[x]$-module. According to the next lemma, $\mathcal{I}$ is generated by some finite set of monomials.

Now consider the ring $\mathcal{L}$ of all constant-coefficient linear partial differential operators in $\mathbb{R}^{m}$, and in it the ideal $\mathcal{L}_{\left\{V_{j}\right\}}$ consisting of all elements which annihilate all polynomials $\sum_{j} p_{j} \circ \pi_{j}$. The map $L \mapsto \sigma(L)$ from $L$ to its symbol is an isomorphism of $\mathcal{L}$ with $\mathbb{C}[x]$, which maps $\mathcal{L}_{\left\{V_{j}\right\}}$ to $\mathcal{I}$. Thus any element $L \in \mathcal{L}_{\left\{V_{j}\right\}}$ may expressed as a finite sum $\sum_{\alpha} \ell_{\alpha} \circ \mathcal{M}_{\alpha}$ where each $\mathcal{M}_{\alpha}$ is a differential monomial which likewise annihilates all $p_{j} \circ \pi_{j}$, and each $\ell_{\alpha}$ is some constant-coefficient linear partial differential operator.

If a polynomial $P$ is nondegenerate relative to $\left\{V_{j}\right\}$, then there exists $L \in \mathcal{L}_{\left\{V_{j}\right\}}$ such that $L(P)$ does not vanish identically. Therefore there exists a differential monomial $\mathcal{M}_{\alpha}$ such that $\mathcal{M}_{\alpha}(P)$ does not vanish identically, so that $P$ is simply nondegenerate.

Lemma 3.6. Let $\mathcal{V}=\cup_{j=1}^{n} \mathcal{V}_{j}$ where each $\mathcal{V}_{j}$ is a linear subspace of $\mathbb{R}^{m}$ of dimension $\kappa$. Suppose that $(m-\kappa) n \leq m$, and that the subspaces $\mathcal{V}_{j}$ lie in general position. Then the ideal $\mathcal{I} \subset \mathbb{R}[x]$ of all polynomials vanishing identically on $\mathcal{V}$ is generated by monomials.

If $\left\{\ell_{i}: 1 \leq i \leq m\right\}$ is some collection of $m$ linearly independent linear functionals from $\mathbb{R}^{m}$ to $\mathbb{R}$, then we may regard the functions $x \mapsto \ell_{i}(x)$ as coordinates on $\mathbb{R}^{m}$. Any polynomial $Q: \mathbb{R}^{m} \rightarrow \mathbb{R}$ can be expressed in a unique way as a finite constant-coefficient linear combination $\sum_{\alpha} c_{\alpha} \ell^{\alpha}$ where $\ell^{\alpha}=\prod_{i} \ell_{i}^{\alpha_{i}}$ and $\alpha=\left(\alpha_{1}, \cdots, \alpha_{m}\right)$. We say that $Q$ is independent of some coordinate $\ell_{j}$ if $c_{\alpha}=0$ whenever $\alpha_{j} \neq 0$.

Proof. Fix linear functions $y_{j, k}(x)$, for $1 \leq j \leq n$ and $1 \leq k \leq m-\kappa$, such that $\mathcal{V}_{j}=\{x$ : $y_{j, k}(x)=0$ for all $\left.1 \leq k \leq m-\kappa\right\}$. The total number of such functions is $(m-\kappa) n \leq m$, and they are linearly independent by the general position hypothesis. If $m=(m-\kappa) n$, we regard these as coordinates on $\mathbb{R}^{m}$; if $m>(m-\kappa) n$ then we take these as being among the coordinates and choose some $m-(m-\kappa) n$ additional linear functions to obtain a full 
coordinate system. In either case this gives a collection of linear functionals $\ell_{i}$ as in the preceding paragraph.

Each of the $(m-\kappa)^{n}$ monomials of the form $\prod_{j=1}^{n} y_{j, k(j)}$ belongs to $\mathcal{I}$. We claim conversely that $\mathcal{I}$ is generated by these monomials.

Let $P \in \mathcal{I}$. Since $P \equiv 0$ on $\mathcal{V}_{1}$, it may be expressed as $P=\sum_{k=1}^{m-\kappa} y_{1, k} r_{k}$ for certain polynomials $r_{k}$, which have the additional property that $r_{k}$ is independent of $y_{1, i}$ for all $i<k$, in the sense discussed above. Consider the restriction of $P$ to the subspace $U=\{x$ : $y_{1, k}(x)=0$ for all $\left.k<m-\kappa\right\}$. Since $P$ vanishes on $\mathcal{V}_{2}$ and thence on $U \cap \mathcal{V}_{2}, y_{1, m-\kappa} r_{m-\kappa}$ must vanish on $U \cap \mathcal{V}_{2}$, and hence, by the general position hypothesis, so must $r_{m-\kappa}$. Since $r_{m-\kappa}$ is independent of $y_{1, i}$ for all $i<k$, this means that $r_{m-\kappa}$ vanishes identically on $\mathcal{V}_{2}$.

Next consider the restriction of $P$ to the subspace $\left\{x: y_{1, k}(x)=0\right.$ for all $\left.k<m-\kappa-1\right\}$. Since $r_{m-\kappa} \equiv 0$ on $\mathcal{V}_{2}$, a repetition of the reasoning of the preceding paragraph demonstrates that $r_{m-\kappa-1} \equiv 0$ on $\mathcal{V}_{2}$. By descending induction on $k$ we eventually conclude that every $r_{k}$ likewise vanishes identically there.

Of course the same applies to $\mathcal{V}_{j}$ for all $j \geq 3$. Thus $P=\sum_{k=1}^{m-\kappa} y_{1, k} r_{k}$ where each $r_{k}$ belongs to the ideal $\tilde{\mathcal{I}}$ of all polynomials vanishing on $\cup_{j=2}^{n} \mathcal{V}_{j}$. By induction on the number $n$ of subspaces $\mathcal{V}_{j}$, we conclude that each $r_{k}$ is generated by monomials belong to $\tilde{\mathcal{I}}$, hence that $P$ is generated by monomial elements of $\mathcal{I}$.

Example. In $\mathbb{R}^{4}$ let $\mathcal{V}_{1}=\left\{x: x_{1}=x_{2}=0\right\}, \mathcal{V}_{2}=\left\{x: x_{3}=x_{4}=0\right\}, \mathcal{V}_{3}=\left\{x: x_{1}-x_{3}=\right.$ $\left.x_{2}-x_{4}=0\right\}$. Thus $m=4, \kappa=2$, and $n=3>m /(m-\kappa)$; the dimensional hypotheses of the preceding two lemmas are not satisfied. The polynomial $p(x)=x_{1} x_{4}-x_{2} x_{3}$ belongs to the associated ideal $\mathcal{I}$. On the other hand, any monomial $\mathcal{M} \in \mathcal{I}$ must have degree at least three. Indeed, if $\mathcal{M}$ has degree two then in order to vanish on $\mathcal{V}_{1} \cup \mathcal{V}_{2}$, it must take the form $\mathcal{M}(x)=\left(a x_{1}+b x_{2}\right)\left(c x_{3}+d x_{4}\right)$. Then $\mathcal{M}\left(x_{1}, x_{2}, x_{1}, x_{2}\right)$ plainly cannot vanish identically on $\mathcal{V}_{3}$ unless $\mathcal{M}$ vanishes identically on $\mathbb{R}^{4}$.

The reasoning underlying Lemma 3.6 and therefore Lemma 3.5 applies more generally. If $\left\{V_{j}\right\}$ is a finite collection of linear subspaces having arbitrary dimensions $\kappa_{j}$, then the conclusion of Lemma 3.5 continues to hold whenever $\sum_{j}\left(m-\kappa_{j}\right) \leq m$. Moreover, if $N<n$ and if $\sum_{j=1}^{N}\left(m-\kappa_{j}\right) \leq m$, then for any homogeneous polynomial $P$ which is nondegenerate relative to $\left\{V_{j}: 1 \leq j \leq n\right\}$, there exists a homogeneous constant-coefficient partial differential operator $\mathcal{L}$ which annihilates $h_{j} \circ \pi_{j}$ for all $j \leq n$ and all distributions $h_{j}$, but which does not annihilate $P$, and which takes the special form $\sum_{\alpha} M_{\alpha} \circ L_{\alpha}$ where $M_{\alpha}, L_{\alpha}$ are likewise homogeneous constant-coefficient partial differential operators, and $L_{\alpha}$ is a monomial $\prod_{j=1}^{N}\left(w_{j, \alpha} \cdot \nabla\right)$ with each vector $w_{j, \alpha}$ orthogonal to $V_{j}$.

\section{The SIMPLY NONDEGENERATE CASE}

In proving (1.2), each function $f_{j}$ may be assumed to be supported in a fixed compact set, the image under $\pi_{j}$ of the support of the cutoff function $\eta$. We will assume this throughout the proofs of Theorems 2.1 and 2.3.

Proof of Theorem 2.3. The proof proceeds by induction on the degree $n$ of multilinearity of $\Lambda_{\lambda}$, and is an adaptation of an argument in [1]. Adopt coordinates in which $V_{n}=\{(y, z) \in$ $\left.\mathbb{R}^{m-1} \times \mathbb{R}^{1}: z=0\right\}$ and hence $\pi_{n}(y, z)=y$. Write

$$
\Lambda_{\lambda}\left(f_{1}, \cdots, f_{n}\right)=\int f_{n}(y)\left(\int e^{i \lambda P(y, z)} \prod_{j=1}^{n-1} f_{j}\left(\pi_{j}(y, z)\right) \eta(y, z) d z\right) d y .
$$


This equals $\left\langle T_{\lambda}\left(f_{1}, \cdots, f_{n-1}\right), \overline{f_{n}}\right\rangle$ for a certain linear operator $T_{\lambda}$, whence

$$
\left|\Lambda_{\lambda}\left(f_{1}, \cdots, f_{n}\right)\right| \leq\left\|f_{n}\right\|_{2}\left\|T_{\lambda}\left(f_{1}, \cdots, f_{n-1}\right)\right\|_{2} \leq C\left\|f_{n}\right\|_{\infty}\left\|T_{\lambda}\left(f_{1}, \cdots, f_{n-1}\right)\right\|_{2} .
$$

Thus it suffices to bound $T_{\lambda}$ as an operator from $L^{\infty} \times \cdots \times L^{\infty}$ to $L^{2}$.

Now $\int\left|T_{\lambda}\left(f_{1}, \cdots, f_{n-1}\right)(y)\right|^{2} d y$ equals

$$
\int_{\mathbb{R}^{m-1}} \iint_{\mathbb{R}^{2}} e^{i \lambda\left(P(y, z)-P\left(y, z^{\prime}\right)\right)} \prod_{j<n} f_{j}\left(\pi_{j}(y, z)\right) \overline{f_{j}}\left(\pi_{j}\left(y, z^{\prime}\right)\right) \eta(y, z) \bar{\eta}\left(y, z^{\prime}\right) d z d z^{\prime} d y .
$$

Define $Q_{\zeta}(y, z)=P(y, z)-P(y, z+\zeta)$, and $\tilde{\eta}_{\zeta}(y, z)=\eta(y, z) \bar{\eta}(y, z+\zeta)$. Likewise define $F_{j}^{\zeta}: V_{j} \rightarrow \mathbb{C}$ so that $F_{j}^{\zeta} \circ \pi_{j}(y, z)=f_{j}\left(\pi_{j}(y, z)\right) \overline{f_{j}}\left(\pi_{j}(y, z+\zeta)\right)$; the right-hand side is a function of $\left(\pi_{j}(y, z), \zeta\right)$ alone because of the linearity of $\pi_{j}$. Of course $\left\|F_{j}^{\zeta}\right\|_{\infty} \leq\left\|f_{j}\right\|_{\infty}^{2}$. With these definitions and the substitutions $x=(y, z), z^{\prime}=z+\zeta$,

$$
\int\left|T_{\lambda}\left(f_{1}, \cdots, f_{n-1}\right)(y)\right|^{2} d y=\int \Lambda_{\lambda}^{\zeta}\left(F_{1}^{\zeta}, \cdots, F_{n-1}^{\zeta}\right) d \zeta
$$

where

$$
\Lambda_{\lambda}^{\zeta}\left(F_{1}^{\zeta}, \cdots, F_{n-1}^{\zeta}\right)=\int_{\mathbb{R}^{m}} e^{i \lambda Q_{\zeta}(x)} \prod_{j=1}^{n-1} F_{j}^{\zeta}\left(\pi_{j}(x)\right) \tilde{\eta}_{\zeta}(x) d x .
$$

The outer integral may be taken over a bounded subset of $\mathbb{R}^{m-\kappa}$, since $\tilde{\eta}_{\zeta} \equiv 0$ when $|\zeta|$ is sufficiently large. Henceforth we assume $\zeta$ to be restricted to such a bounded set.

For each $\zeta$, consider the polynomial phase $Q_{\zeta}$. We may assume that $|\lambda| \geq 1$, since (1.2) holds trivially otherwise. It is given as a hypothesis that there exists a differential operator of the form $L=\prod_{j=1}^{n}\left(w_{j} \cdot \nabla\right)$, with each $w_{j} \in V_{j}^{\perp}$, such that $\sup _{|x| \leq 1}|L(P)(x)| \geq c>0$. Let $L^{\prime}=\prod_{j<n}\left(w_{j} \cdot \nabla\right)$. For any $\rho \in(0,1)$ define

$$
E_{\rho}=\left\{\zeta: \max _{|x| \leq 1}\left|L^{\prime} Q_{\zeta}(x)\right| \leq \rho\right\}
$$

For any $\zeta \notin E_{\rho}$, write $\lambda Q_{\zeta}=(\lambda \rho) \tilde{Q}$ where $\tilde{Q}=\rho^{-1} Q_{\zeta}$. Then by the induction hypothesis, there exist $C, \varepsilon^{\prime} \in \mathbb{R}^{+}$such that for all $\zeta \notin E_{\rho}$,

$$
\left|\Lambda_{\lambda}^{\zeta}\left(F_{1}^{\zeta}, \cdots, F_{n-1}^{\zeta}\right)\right| \leq C(1+|\lambda| \rho)^{-\varepsilon^{\prime}} \prod_{j<n}\left\|F_{j}^{\zeta}\right\|_{\infty} \leq C(1+|\lambda| \rho)^{-\varepsilon^{\prime}} \prod_{j<n}\left\|f_{j}\right\|_{\infty}^{2} .
$$

The same bound holds for the integral over all $\zeta \notin E_{\rho}$, since $\zeta$ is confined to a bounded set.

For $\zeta \in E_{\rho}$ there is the trivial estimate

$$
\left|\Lambda_{\lambda}^{\zeta}\left(F_{1}^{\zeta}, \cdots, F_{n-1}^{\zeta}\right)\right| \leq C \prod_{j<n}\left\|F_{j}^{\zeta}\right\|_{\infty}
$$

SO

$$
\int_{E_{\rho}}\left|\Lambda_{\lambda}^{\zeta}\left(F_{1}^{\zeta}, \cdots, F_{n-1}^{\zeta}\right)\right| d \zeta \leq C\left|E_{\rho}\right| \prod_{j<n}\left\|f_{j}\right\|_{L^{\infty}}^{2}
$$

Now

$$
\left|E_{\rho}\right| \leq C \rho^{\delta} \text { for some } \delta>0 \text { and } C<\infty .
$$

Indeed, by hypothesis $\sup _{(x, \zeta)}\left|\partial_{\zeta}\left(L^{\prime} Q_{\zeta}(x)\right)\right| \geq c>0$, and as is well known, this implies that if $(x, \zeta)$ is restricted to any fixed bounded set, then

$$
\left|\left\{(x, \zeta):\left|L^{\prime} Q_{\zeta}(x)\right| \leq r\right\}\right| \leq C r^{a}
$$


for some $a>0$ where $C, a$ depend only on $c$ and on an upper bound for the degree of $Q$ as a polynomial in $(x, \zeta)$. In particular, so long as $x, \zeta$ are restricted to lie in any fixed bounded set,

Thus in all

$$
\left|\left\{\zeta: \max _{x}\left|L^{\prime} Q_{\zeta}(x)\right| \leq r\right\}\right| \leq C r^{a} .
$$

$$
\int\left|\Lambda_{\lambda}^{\zeta}\left(F_{1}^{\zeta}, \cdots, F_{n-1}^{\zeta}\right)\right| d \zeta \leq C\left[(|\lambda| \rho)^{-\varepsilon^{\prime}}+\rho^{\delta}\right] \prod_{j<n}\left\|f_{j}\right\|_{\infty}^{2} .
$$

Choosing $\rho=|\lambda|^{-c}$ for any fixed $c \in(0,1)$ yields the desired bound.

\section{THE POWER DECAY PROPERTY FOR $\kappa=1$}

This section is devoted to the proof of Theorem 2.1. The proof turns on a concept related to a notion of uniformity employed by Gowers [2]. Let $d \geq 1$, and fix a bounded ball $B \subset \mathbb{R}^{m}$. Let $\tau>0$ be a small quantity to be chosen below, let $|\lambda| \geq 1$, and consider any function $f \in L^{2}\left(\mathbb{R}^{m}\right)$ supported in $B$.

Definition 5.1. $f$ is $\lambda$-nonuniform if there exist a polynomial $q$ of degree $\leq d$ and a scalar $c$ such that

$$
\left\|f-c e^{i q}\right\|_{L^{2}(B)} \leq\left(1-|\lambda|^{-\tau}\right)\|f\|_{L^{2}} .
$$

Otherwise $f$ is said to be $\lambda$-uniform.

This notion depends on the parameters $d, \tau$. So long as they remain fixed there exists $C<\infty$, depending also on $B$, such that any $\lambda$-uniform function $f \in L^{2}(B)$ satisfies favorable bounds for generalized Fourier coefficients:

$$
\left|\int f(t) e^{-i q(t)} d t\right| \leq C|\lambda|^{-\tau / 2}\|f\|_{L^{2}(B)}
$$

uniformly for all real-valued polynomials $q$ of degree $\leq d$. Indeed, $f$ could otherwise be decomposed in $L^{2}(B)$ into its projection onto $e^{i q}$ plus an orthogonal vector, implying (5.1).

The proof of Theorem 2.1 will proceed by induction on $n$, the number of subspaces $\left\{V_{j}\right\}$. The inductive step enters in the following way: If $f_{1}=e^{i p}$ for some polynomial $p$, then $\Lambda_{\lambda}\left(e^{i p}, f_{2}, \cdots, f_{n}\right)=\int e^{i \tilde{P}(x)} \prod_{j=2}^{n} f_{j}\left(\pi_{j}(x)\right) \eta(x) d x$ where $\tilde{P}=P+p \circ \pi_{1}$. With $p$ held fixed, this may be regarded as an $(n-1)$-multilinear operator acting on $\left(f_{2}, \cdots, f_{n}\right)$, with the new phase $\tilde{P}$ and the smaller collection of subspaces $\left\{V_{j}: 2 \leq j \leq n\right\}$. $\tilde{P}$ is nondegenerate relative to this subcollection; moreover, this nondegeneracy is uniform as $p$ varies over all polynomials of uniformly bounded degrees. Thus it is a consequence of the inductive hypothesis that

$$
\left|\Lambda_{\lambda}\left(e^{i p}, f_{2}, \cdots, f_{n}\right)\right| \leq C|\lambda|^{-\varepsilon}
$$

provided that $\left\|f_{j}\right\|_{2} \leq 1$ for all $2 \leq j \leq n$, uniformly for all polynomials $p$ of uniformly bounded degrees.

Proof of Theorem 2.1. Given the cutoff function $\eta$, there exist intervals $B_{j}$ of finite lengths in $\mathbb{R}^{1}$ such that $\Lambda_{\lambda}\left(f_{1}, \cdots, f_{n}\right)$ depends only on the restriction of each $f_{j}$ to $B_{j}$, so henceforth we assume $f_{j}$ to be supported in $B_{j}$. Thus $\left\|f_{j}\right\|_{L^{2}}$ equals $\left\|f_{j}\right\|_{L^{2}\left(B_{j}\right)}$.

We may assume henceforth ${ }^{1}$ that $n$ is strictly larger than $m$, since the theorem is already known in a more precise form [8],[9],[10] for the case $n=m$. Let $e_{1}$ be a unit vector

\footnotetext{
${ }^{1}$ Our proof can be adapted to the simpler case $n \leq m$ as well.
} 
orthogonal to the span of $\left\{V_{j}: 2 \leq j \leq m\right\}$. Since the subspaces $\left\{V_{j}: 2 \leq j \leq m\right\}$ span a space of codimension one, $e_{1}$ is uniquely determined modulo multiplication by -1 , and it cannot be orthogonal to $V_{1}$ because of the general position hypothesis.

Likewise choose some unit vector $e_{2}$ orthogonal to $\operatorname{span}\left(\left\{V_{j}: j>m\right\}\right)$, and not orthogonal to $V_{1} \cdot \cup_{j>m} V_{j}$ spans a space of dimension $n-m<m$ by the assumption that $n<2 m$ and the general position hypothesis, and the only way that all unit vectors in its orthocomplement could be forced to be orthogonal to $V_{1}$ is if $V_{1}$ were to be contained in $\operatorname{span}\left(\left\{V_{j}: j>m\right\}\right)$. But this cannot happen, again by general position and the restriction $n-m<m$. Thus there exists at least one vector $e_{2}$ with the required properties.

$e_{2}$ cannot be orthogonal to $\operatorname{span}\left(\left\{V_{j}: 2 \leq j \leq m\right\}\right)$, since $\cup_{j=2}^{n} V_{j}$ spans $\mathbb{R}^{m}$ by the general position assumption $(n>m)$. $e_{1}$ cannot be orthogonal to span $\left(\left\{V_{j}: j>m\right\}\right)$, since then it would be orthogonal to $\cup_{j \geq 2} V_{j}$, which we have just seen to be impossible. Thus $e_{2}$ is automatically linearly independent of $e_{1}$.

Given $\left\{V_{j}\right\}, P$, a cutoff function $\eta$, and $\lambda$, define $A(\lambda)$ to be the best constant (that is, the infimum of all admissible constants) in the inequality

$$
\left|\Lambda_{\lambda}\left(f_{1}, \cdots, f_{n}\right)\right| \leq A(\lambda) \prod_{j}\left\|f_{j}\right\|_{L^{2}} .
$$

As noted before the statement of Theorem 2.1, the hypotheses of general position and $n \leq 2 m$ ensure that $A(\lambda)$ is finite for all $\lambda$. We will assume that $\left\|f_{j}\right\|_{L^{2}} \leq 1$ for all $j$, and that $|\lambda|$ exceeds some sufficiently large constant. To prove the theorem it suffices to obtain a bound of $C|\lambda|^{-\varepsilon}$ under these additional hypotheses.

The analysis of $\Lambda_{\lambda}\left(f_{1}, \cdots, f_{n}\right)$ is divided into two cases, depending on whether or not $f_{1}$ is $\lambda$-uniform. If it is not, let $f=f_{1}, c, q$ satisfy (5.1). Then

$$
\left|\Lambda_{\lambda}\left(f_{1}-c e^{i q}, f_{2}, \cdots, f_{n}\right)\right| \leq A(\lambda)\left(1-|\lambda|^{-\tau}\right) .
$$

Moreover $|c|$ is majorized by an absolute constant since $\left\|f_{1}\right\|_{L^{2}}=1$ and $e^{i q}$ is unimodular, so by the inductive hypothesis (5.3)

$$
\left|\Lambda_{\lambda}\left(c e^{i q}, f_{2}, \cdots, f_{n}\right)\right| \leq C\left|\Lambda_{\lambda}\left(e^{i q}, f_{2}, \cdots, f_{n}\right)\right| \leq C|\lambda|^{-\sigma}
$$

for certain $C, \sigma \in(0, \infty)$; this bound holds uniformly provided that $P$ varies over a set of uniformly nondegenerate polynomials. Combining the two terms yields

$$
\left|\Lambda_{\lambda}\left(f_{1}, \cdots, f_{n}\right)\right| \leq A(\lambda)\left(1-|\lambda|^{-\tau}\right)+|\lambda|^{-\sigma}
$$

provided that $\left\|f_{j}\right\|_{L^{2}} \leq 1$ for all $j$, and that $f_{1}$ is $\lambda$-nonuniform.

Suppose next that $f_{1}$ is $\lambda$-uniform. Adopt coordinates $(t, y) \in \mathbb{R}^{2} \times \mathbb{R}^{m-2}$ where $\mathbb{R}^{2}$ is the span of $e_{1}, e_{2}$ and $\mathbb{R}^{m-2}$ is its orthocomplement, by writing $x=t_{1} e_{1}+t_{2} e_{2}+y$. Let $P^{y}(t)=P(t, y)$. Define

$$
F_{1}^{y}\left(t_{2}\right)=\prod_{j=2}^{m} f_{j} \circ \pi_{j}(t, y) ;
$$

the right-hand side is independent of $t_{1}$ since $e_{1}$ is orthogonal to $V_{j}$ for all $2 \leq j \leq m$. Likewise define

$$
F_{2}^{y}\left(t_{1}\right)=\prod_{j=m+1}^{n} f_{j} \circ \pi_{j}(t, y),
$$

which is independent of $t_{2}$. In the same way, since neither $e_{1}$ nor $e_{2}$ is orthogonal to $V_{1}$, $f_{1} \circ \pi_{1}(t, y)$ depends for each $y$ only on a certain projection $\pi(t)$ of $t \in \mathbb{R}^{2}$ onto $\mathbb{R}^{1}$, so we 
may write

$$
G^{y}(\pi(t))=f_{1} \circ \pi_{1}(t, y) .
$$

The general position hypothesis, the hypothesis $n \leq 2 m$, and Fubini's theorem together imply that

$$
\begin{aligned}
& \int\left\|F_{1}^{y}\right\|_{L^{2}}^{2}\left\|G^{y}\right\|_{L^{2}}^{2} d y=C \prod_{j=1}^{m}\left\|f_{j}\right\|_{L^{2}}^{2} \\
& \int\left\|F_{2}^{y}\right\|_{L^{2}}^{2} d y=C \prod_{j=m+1}^{n}\left\|f_{j}\right\|_{L^{2}}^{2}
\end{aligned}
$$

for a certain positive constant $C$, and consequently

$$
\int\left\|F_{1}^{y}\right\|_{L^{2}}\left\|F_{2}^{y}\right\|_{L^{2}}\left\|G^{y}\right\|_{L^{2}} d y \leq C \prod_{j=1}^{n}\left\|f_{j}\right\|_{L^{2}}
$$

by Cauchy-Schwarz.

For each $y \in \mathbb{R}^{m-2}$ consider

$$
\Lambda_{\lambda}^{y}=\int_{\mathbb{R}^{2}} e^{i \lambda P^{y}(t)} F_{1}^{y}\left(t_{2}\right) F_{2}^{y}\left(t_{1}\right) G^{y}(\pi(t)) \eta(t, y) d t .
$$

Define the set $\mathcal{B} \subset \mathbb{R}^{m-2}$ of bad parameters to be the set of all $y$ for which $P^{y}$ may be decomposed as

$$
P^{y}(t)=Q_{1}\left(t_{1}\right)+Q_{2}\left(t_{2}\right)+Q_{3}(\pi(t))+R(t)
$$

where $Q_{j}$ are real-valued polynomials of degrees $\leq d$ on $\mathbb{R}^{1}$ for $j=1,2, t_{j}$ is a certain linear function of $t, R: \mathbb{R}^{2} \rightarrow \mathbb{R}$ is likewise a real-valued polynomial of degree $\leq d$, and

$$
\|R\| \leq|\lambda|^{-\rho}
$$

that is, $P^{y}$ has small norm in the quotient space of polynomials modulo degenerate polynomials, relative to the three projections $t \mapsto t_{1}, t_{2}, \pi(t)$. Here $\|\cdot\|$ denotes any fixed norm on the vector space of all polynomials of degree $\leq d$, and $\rho \in(0,1)$ is another parameter to be specified. If $y \notin \mathcal{B}$ then $|\lambda|^{\rho} P^{y}$ is at least a fixed positive distance from the span of all polynomials $Q_{1}\left(t_{1}\right)+Q_{2}\left(t_{2}\right)+Q_{3}(\pi(t))$, so we may apply Theorem 2.3 with $n=3, m=2$, and the phase $|\lambda|^{\rho} P^{y}$, to obtain

$$
\left|\Lambda_{\lambda}^{y}\right| \leq C\left(|\lambda|^{1-\rho}\right)^{-\tilde{\rho}}\left\|F_{1}^{y}\right\|_{L^{2}}\left\|F_{2}^{y}\right\|_{L^{2}}\left\|G^{y}\right\|_{L^{2}} \text { for some } C, \tilde{\rho} \in \mathbb{R}^{+} .
$$

This together with (5.6) implies that

$$
\int_{y \notin \mathcal{B}}\left|\Lambda_{\lambda}^{y}\right| d y \leq C|\lambda|^{-(1-\rho) \tilde{\rho}}
$$

as desired.

Bad parameters cannot be handled in this way. Nor is it true that the set of bad parameters has measure $O\left(|\lambda|^{-\delta}\right)$ for some $\delta>0$; it could happen that every parameter is bad. Nonetheless we claim that if the exponent $\rho$ appearing in the definition of bad parameters is chosen to be sufficiently small, then there exists $\varepsilon>0$ such that uniformly for all $y \in \mathcal{B}$,

$$
\left|\Lambda_{\lambda}^{y}\right| \leq C|\lambda|^{-\varepsilon}\left\|F_{1}^{y}\right\|_{L^{2}}\left\|F_{2}^{y}\right\|_{L^{2}}\left\|G^{y}\right\|_{L^{2}}
$$


which by (5.6) again implies the desired bound. To verify this fix $y \in \mathcal{B}$, and let $P^{y}(t)=$ $Q_{1}\left(t_{1}\right)+Q_{2}\left(t_{2}\right)+Q_{3}(\pi(t))+R(t)$ as above. Write $\pi(t)=c_{1} t_{1}+c_{2} t_{2}$ where $c_{1}, c_{2}$ are both nonzero. Introduce the functions

$$
\begin{aligned}
\tilde{F}_{1}\left(t_{2}\right) & =F_{1}^{y}\left(t_{2}\right) e^{i \lambda Q_{2}\left(t_{2}\right)}, \\
\tilde{F}_{2}\left(t_{1}\right) & =F_{2}^{y}\left(t_{1}\right) e^{i \lambda Q_{1}\left(t_{1}\right)}, \\
\tilde{G}(s) & =G^{y}(s) e^{i \lambda Q_{3}(s)}, \\
\zeta(t) & =\eta(t, y) e^{i \lambda R(t)} .
\end{aligned}
$$

Since $\lambda R$ is a polynomial of bounded degree which is $O\left(|\lambda|^{1-\rho}\right)$ on the support of $\zeta$, the same holds for all its derivatives and therefore

$$
|\widehat{\zeta}(\xi)| \leq C_{N}|\lambda|^{(1-\rho) N}(1+|\xi|)^{-N} \text { for all } \xi \text { and all } N \text {. }
$$

By Fourier inversion,

$$
\Lambda_{\lambda}^{y}=c \int_{\mathbb{R}^{3}} \widehat{\widetilde{G}}\left(\xi_{0}\right) \widehat{\tilde{F}_{1}}\left(-c_{2} \xi_{0}-\xi_{2}\right) \widehat{\tilde{F}_{2}}\left(-c_{1} \xi_{0}-\xi_{1}\right) \widehat{\zeta}\left(\xi_{1}, \xi_{2}\right) d \xi_{0} d \xi_{1} d \xi_{2}
$$

From this representation, the bound (5.9) with say $N=4$, and Plancherel's theorem, it follows that

$$
\left|\Lambda_{\lambda}^{y}\right| \leq C|\lambda|^{4(1-\rho)}\|\widehat{\tilde{G}}\|_{L^{\infty}}\left\|\tilde{F}_{1}\right\|_{L^{2}}\left\|\tilde{F}_{2}\right\|_{L^{2}}
$$

Here $\left\|\tilde{F}_{i}\right\|_{L^{2}}=\left\|F_{i}^{y}\right\|_{L^{2}}$. $\quad \tilde{G}$ is obtained from $f_{1}$ by composing with a fixed linear mapping, translating by an arbitrary amount, and multiplying with $e^{i q}$ for some real-valued polynomial $q$ of degree $\leq d$. Therefore the uniformity condition (5.2) for $f_{1}$ implies that

$$
\|\widehat{\widetilde{G}}\|_{L^{\infty}} \leq C|\lambda|^{-\tau}
$$

for some $C, \tau \in \mathbb{R}^{+}$independent of $y$. Thus if $f_{1}$ is $\lambda$-uniform then

$$
\left|\Lambda_{\lambda}^{y}\right| \leq C|\lambda|^{4(1-\rho)-\tau}\left\|F_{1}^{y}\right\|_{L^{2}}\left\|F_{2}^{y}\right\|_{L^{2}} \leq C|\lambda|^{4(1-\rho)-\tau} .
$$

The parameter $\rho \in(0,1)$ is at our disposal; we choose $\rho<1$ sufficiently close to 1 such that $4(1-\rho)-\tau=-\varepsilon$ is strictly negative. This completes the analysis of the case where $f_{1}$ is $\lambda$-uniform.

Combined with (5.5) and (5.7), this proves that

$$
\left.A(\lambda) \leq C \max \left(|\lambda|^{-\varepsilon},|\lambda|^{-(1-\rho) \tilde{\rho}}, A(\lambda)\left(1-|\lambda|^{-\tau}\right)+|\lambda|^{-\sigma}\right)\right) .
$$

Since $A(\lambda)$ is certainly finite, this implies that it is majorized by a constant times some negative power of $|\lambda|$, as was to be proved.

\section{Oscillatory Singular INTEGRAL OPERATORS}

In this section we combine the results on nonsingular oscillatory integral operators proved above with the currently existing theory of multilinear Calderón-Zygmund singular integral operators to prove Theorems 2.5 and 2.6. Let $d, k, n \geq 1$, and let $K$ be a Calderón-Zygmund distribution in $\mathbb{R}^{k}$. Thus $K$ is a tempered distribution which agrees with a Lipschitz function on $\mathbb{R}^{k} \backslash\{0\}$, and $K(r y) \equiv r^{-k} y$ in the sense of distributions. For $1 \leq j \leq n$ let $\ell_{j}: \mathbb{R}^{k} \rightarrow \mathbb{R}^{d}$ be a linear mapping, and suppose that the intersection of the nullspaces of all the $\ell_{j}$ is $\{0\}$. Consider the multilinear operators

$$
T\left(f_{1}, \cdots, f_{n}\right)(x)=\left\langle K, \prod_{j=1}^{n} f_{j}\left(x+\ell_{j}(\cdot)\right)\right\rangle
$$


where $x \in \mathbb{R}^{d}, f_{j} \in C_{0}^{\infty}\left(\mathbb{R}^{d}\right)$, and the pairing is that of the distribution $K$ with the test function $y \mapsto \prod_{j=1}^{n} f_{j}\left(x+\ell_{j}(y)\right)$. To any real-valued polynomial $P(x, y)$ defined on $\mathbb{R}^{d+k}$ is associated the operator

$$
T_{P}\left(f_{1}, \cdots, f_{n}\right)(x)=\left\langle e^{i P(x, \cdot)} K, \prod_{j=1}^{n} f_{j}\left(x+\ell_{j}(\cdot)\right)\right\rangle,
$$

which is again well-defined when each $f_{j} \in C_{0}^{\infty}\left(\mathbb{R}^{d}\right)$.

If $q^{-1}=\sum_{j} p_{j}^{-1}$ then a necessary condition for $T_{P}$ to map $\otimes_{j} L^{p_{j}}$ boundedly to $L^{q}$ is that $T$ should do so. It is not known under what conditions $T$ acts boundedly on Lebesgue spaces, but we can assert a conditional result, generalizing Theorem 2.6. Set $\ell_{0}(y) \equiv 0$ so that $x+\ell_{0}(y) \equiv x$, and define $\pi_{0}(x, y)=x$. Define $V_{j} \subset \mathbb{R}^{d+k}$ for each $j \geq 1$ to be the orthocomplement of the nullspace of the mapping $(x, y) \mapsto x+\ell_{j}(y)$, and let $\pi_{j}$ be the orthogonal projection onto $V_{j}$. Then $f_{j}\left(x+\ell_{j}(y)\right)=\tilde{f}_{j}\left(\pi_{j}(x, y)\right)$ where $\left\|\tilde{f}_{j}\right\|_{p_{j}}$ is a constant multiple of $\left\|f_{j}\right\|_{p_{j}}$.

Theorem 6.1. Let $d, n, K, T,\left\{\ell_{j}\right\}$ be as above. Suppose that $T$ maps $\otimes_{j=1}^{n} L^{p_{j}}\left(\mathbb{R}^{d}\right)$ boundedly to $L^{q}\left(\mathbb{R}^{d}\right)$ for some exponents $1<p_{j}<\infty$ and $0<q<\infty$ satisfying $q^{-1}=\sum_{j} p_{j}^{-1}$. Suppose furthermore that the collection $\left\{V_{j}: 0 \leq j \leq n\right\}$ of orthocomplements of the nullspaces of $\ell_{j}$ has the uniform decay property. Then for any real-valued polynomial $P$, $T_{P}$ maps $\otimes_{j=1}^{n} L^{p_{j}}$ boundedly to $L^{q}$. Moreover for any $D$ there exists $C_{D}<\infty$ such that $\left\|T_{P}\left(f_{1}, \cdots, f_{n}\right)\right\|_{q} \leq C_{D} B \prod_{j}\left\|f_{j}\right\|_{p_{j}}$ uniformly for all real-valued polynomials $P$ of degrees $\leq D$, where $B$ is the operator norm of $T$ from $\otimes_{j} L^{p_{j}}$ to $L^{q}$.

If $\left\{V_{j}\right\}$ merely has the uniform decay property for all polynomials of degrees $\leq D$, then the conclusion of the theorem holds for polynomials of degrees $\leq D$.

A sufficient condition for $T$ to be bounded was established in [6]. Combining it with Theorem 6.1 gives Theorem 2.6, and its special case Theorem 2.5.

The proof of Theorem 6.1 is little different from its linear prototype [9]. One step in the proof requires passing to associated truncated operators. This can be done quite generally by means of the following lemma, versions of which have appeared elsewhere in the literature.

Lemma 6.2. Let $k \geq \kappa \geq 1$ and $n \geq 0$ be integers. For $1 \leq j \leq n$ let $\ell_{j}: \mathbb{R}^{k} \rightarrow \mathbb{R}^{\kappa}$ be linear mappings, the intersection of all of whose nullspaces is $\{0\}$. Let $p_{j} \in[1, \infty]$ and let $q^{-1}=\sum_{j} p_{j}^{-1}$.

Let $\eta \in C_{0}^{\infty}\left(\mathbb{R}^{m}\right)$ have compact support. There exists $A<\infty$, depending only on a sufficiently high order $C^{M}$ norm of $\eta$ and the support of $\eta$, with the following property. Let $\mathcal{K}$ be any tempered distribution in $\mathbb{R}^{k}$, and suppose there exists $C<\infty$ such that

$$
\left\|\left\langle\mathcal{K}, \prod_{j=1}^{n} f_{j}\left(x+\ell_{j}(\cdot)\right)\right\rangle\right\|_{L^{q}} \leq C \prod_{j}\left\|f_{j}\right\|_{L^{p_{j}}} \text { for all } f_{j} \in C_{0}^{\infty}\left(\mathbb{R}^{\kappa}\right) .
$$

Then

$$
\left\|\left\langle\eta \mathcal{K}, \prod_{j=1}^{n} f_{j}\left(x+\ell_{j}(\cdot)\right)\right\rangle\right\|_{L^{q}} \leq A C \prod_{j}\left\|f_{j}\right\|_{L^{p_{j}}} \text { for all } f_{j} \in C_{0}^{\infty}\left(\mathbb{R}^{\kappa}\right) .
$$

As in Theorem 6.1, $q$ is permitted to be strictly less than one. Here $\langle\mathcal{K}, \cdot\rangle$ denotes the pairing of distribution with test function; the hypothesis that the intersection of the nullspaces is trivial guarantees that $\prod_{j=1}^{n} f_{j} \circ \ell_{j}$ is compactly supported. 
Proof. Write $\mathcal{T}_{\mathcal{K}}\left(f_{1}, \cdots, f_{n}\right)(x)=\left\langle\mathcal{K}, \prod_{j} f_{j}\left(x+\ell_{j}(\cdot)\right)\right\rangle$. Let $h \in C^{\infty}\left(\mathbb{R}^{\kappa}\right)$ satisfy $\sum_{\nu \in \mathbb{Z}^{\kappa}} h(x-$ $\nu)=1$ for all $x \in \mathbb{R}^{\kappa}$. Then

$$
\mathcal{T}_{\eta \mathcal{K}}\left(f_{1}, \cdots, f_{n}\right)(x)=\sum_{\left(\nu_{0}, \cdots, \nu_{n}\right) \in \mathbb{Z}^{(n+1) \kappa}} h\left(x-\nu_{0}\right)\left\langle\mathcal{K} \eta, \prod_{j=1}^{n} f_{j}\left(x+\ell_{j}(\cdot)\right) h\left(x+\ell_{j}(\cdot)-\nu_{j}\right)\right\rangle .
$$

Because $\eta$ has compact support, there exists $C_{0}<\infty$ such that $h\left(x-\nu_{0}\right)\left\langle\mathcal{K} \eta, \prod_{j=1}^{n} f_{j}(x+\right.$ $\left.\left.\ell_{j}(\cdot)\right) h\left(x+\ell_{j}(\cdot)-\nu_{j}\right)\right\rangle \equiv 0$ unless $\left|\nu_{j}-\nu_{0}\right| \leq C_{0}$ for all $1 \leq j \leq n$. We write $\sum_{\nu}^{*}$ to denote the summation over all $\nu=\left(\nu_{0}, \cdots, \nu_{n}\right) \in \mathbb{Z}^{(n+1) \kappa}$ satisfying this restriction. Thus

$$
\mathcal{T}_{\eta \mathcal{K}}\left(f_{1}, \cdots, f_{n}\right)(x)=\sum_{\nu}^{*} h\left(x-\nu_{0}\right)\left\langle\mathcal{K} \eta, \prod_{j=1}^{n} f_{j}\left(x+\ell_{j}(\cdot)\right) h\left(x+\ell_{j}(\cdot)-\nu_{j}\right)\right\rangle .
$$

There exists $C_{1}<\infty$ such that for any $\nu \in \mathbb{Z}^{(n+1) \kappa}$ satisfying $\left|\nu_{j}-\nu_{0}\right| \leq C_{0}$ for all $j \geq 1$, and for any point $(x, y) \in \mathbb{R}^{\kappa+m}$ such that $h\left(x-\nu_{0}\right) \neq 0$ and $h\left(x+\ell_{j}(y)-\nu_{j}\right) \neq 0$ for all $j \geq 1$, necessarily $|y| \leq C_{1}$. This follows directly from the assumption that the intersection of the nullspaces of all the linear mappings $\ell_{j}$ equals $\{0\}$. Define $\tilde{\eta}: \mathbb{R}^{k} \rightarrow \mathbb{R}$ to be the unique function that is periodic with periodic $2 C_{1}$ with respect to each coordinate in $\mathbb{R}^{m}$, and that satisfies $\tilde{\eta}(x)=\eta(x)$ whenever $x=\left(x_{1}, \cdots, x_{k}\right)$ with every $\left|x_{j}\right| \leq C_{1}$. Then $\tilde{\eta} \in C^{\infty}$. We may replace $\eta$ by $\tilde{\eta}$ :

$$
\mathcal{T}_{\eta \mathcal{K}}\left(f_{1}, \cdots, f_{n}\right)(x)=\sum_{\nu}^{*} h\left(x-\nu_{0}\right)\left\langle\mathcal{K} \tilde{\eta}, \prod_{j=1}^{n} f_{j}\left(x+\ell_{j}(\cdot)\right) h\left(x+\ell_{j}(\cdot)-\nu_{j}\right)\right\rangle .
$$

By dilating all variables by $\pi / C_{1}$ we may assume that $C_{1}=\pi$, so that $\tilde{\eta}$ is $2 \pi$-periodic with respect to each coordinate and hence $\tilde{\eta}(z)=\sum_{\mu \in \mathbb{Z}^{k}} a_{\mu} e^{i \mu \cdot z}$ where $a_{\mu}=O\left(|\mu|^{-N}\right)$ as $|\mu| \rightarrow \infty$, for all finite $N$. Inserting this into the preceding expression gives

$$
\mathcal{T}_{\eta \mathcal{K}}\left(f_{1}, \cdots, f_{n}\right)(x)=\sum_{\mu \in \mathbb{Z}^{k}} \sum_{\nu}^{*} h\left(x-\nu_{0}\right)\left\langle\mathcal{K}, e^{i \mu \cdot y} \prod_{j=1}^{n} f_{j}\left(x+\ell_{j}(y)\right) h\left(x+\ell_{j}(y)-\nu_{j}\right)\right\rangle_{y},
$$

where this notation indicates that the pairing is between the distribution $\mathcal{K}$ and the function $\mathbb{R}^{k} \ni y \mapsto e^{i \mu \cdot y} \prod_{j=1}^{n} f_{j}\left(x+\ell_{j}(y)\right) h\left(x+\ell_{j}(y)-\nu_{j}\right)$

Since the intersection of the nullspaces of $\left\{\ell_{j}\right\}$ is trivial, there exist linear mappings $L_{j}: \mathbb{R}^{\kappa} \rightarrow \mathbb{R}^{k}$ such that $y \equiv \sum_{j=1}^{n} L_{j} \circ \ell_{j}(y)$ for all $y \in \mathbb{R}^{k}$. Therefore

$$
e^{i \mu \cdot y}=e^{-i \mu \cdot \sum_{j} L_{j}(x)} \prod_{j=1}^{n} e^{i \mu \cdot L_{j}\left(x+\ell_{j}(y)\right)} .
$$

Inserting this into the preceding expression gives

$$
\mathcal{T}_{\eta \mathcal{K}}\left(f_{1}, \cdots, f_{n}\right)(x)=\sum_{\mu \in \mathbb{Z}^{k}} a_{\mu} \sum_{\nu}^{*} e^{-i \mu \cdot \sum_{j} L_{j}(x)} h\left(x-\nu_{0}\right)\left\langle\mathcal{K}, \prod_{j=1}^{n} \tilde{f}_{j, \mu, \nu_{j}}\left(x+\ell_{j}(\cdot)\right)\right\rangle
$$

where $\tilde{f}_{j, \mu, \nu_{j}}(z)=f(z) e^{i \mu \cdot L_{j}(z)} h\left(z-\nu_{j}\right)$.

By hypothesis,

$$
\left\|\left\langle\mathcal{K}, \prod_{j=1}^{n} \tilde{f}_{j, \mu, \nu_{j}}\left(x+\ell_{j}(\cdot)\right)\right\rangle\right\|_{q} \leq C \prod_{j=1}^{n}\left\|\tilde{f}_{j, \mu, \nu_{j}}\right\|_{p_{j}}=C \prod_{j=1}^{n}\left\|f_{j} h\left(\cdot-\nu_{j}\right)\right\|_{p_{j}} .
$$


If $q \geq 1$ then by the triangle inequality, the fact that no point belongs to the support of $h\left(\cdot-\nu_{0}\right)$ for more than a uniformly bounded number of indices $\nu_{0}$, and the finiteness of $\sum_{\mu}\left|a_{\mu}\right|$

$$
\left\|\mathcal{T}_{\eta \mathcal{K}}\left(f_{1}, \cdots, f_{n}\right)(x)\right\|_{q}^{q} \leq C \sum_{\nu}^{*} \prod_{j=1}^{n}\left\|f_{j} h\left(\cdot-\nu_{j}\right)\right\|_{p_{j}}^{q} .
$$

Fix any $\left(v_{1}, \cdots, v_{n}\right) \in\left(\mathbb{Z}^{\kappa}\right)^{n}$. Then

$$
\sum_{\nu_{0} \in \mathbb{Z}^{\kappa}} \prod_{j=1}^{n}\left\|f_{j} h\left(\cdot-\nu_{0}-v_{j}\right)\right\|_{L^{p_{j}}}^{q} \leq \prod_{j=1}^{n}\left(\sum_{\nu_{0} \in \mathbb{Z}^{\kappa}}\left\|f_{j} h\left(\cdot-\nu_{0}-v_{j}\right)\right\|_{L^{p_{j}}}^{p_{j}}\right)^{q / p_{j}}
$$

by Hölder's inequality with exponents $p_{1} / q, \cdots, p_{n} / q$. The right-hand side does not exceed $C \prod_{j=1}^{n}\left\|f_{j}\right\|_{p_{j}}^{q / p_{j}}$. Now because the sum $\sum_{\nu}^{*}$ is subject to the restriction that $\left|\nu_{j}-\nu_{0}\right| \leq C_{0}$ for all $j \geq 1$, summming over an appropriate finite set of values of $\left(v_{1}, \cdots, v_{n}\right)$ gives a majorization for the total. This completes the proof for $q \geq 1$.

For $0<q<1$ we use instead the modified triangle inequality $\left|\sum_{i} b_{i}\right|^{q} \leq \sum_{i}\left|b_{i}\right|^{q}$ together with the fact that $\left|a_{\mu}\right|=O\left(|\mu|^{-N}\right)$ for all $N$ as $|\mu| \rightarrow \infty$. If $N$ is taken to be sufficiently large, depending on $q$ and on $k$, then this bound permits a repetition of the above reasoning.

Proof of Theorem 6.1. Let subspaces $V_{j}$, a degree $D \geq 1$, and a polynomial $P$ of degree $\leq D$ be given. We proceed by induction on $D$, the result for $D=0$ being given as a hypothesis.

Decompose $P=P_{D}+R$ where $R$ has degree $<D$ and $P_{D}$ is homogeneous of degree $D$. If $P_{D}$ is degenerate then by decomposing $P_{D}=\sum_{j} q_{j}\left(x+\ell_{j}(y)\right)$ for certain real-valued polynomials $q_{j}$, we may rewrite $\mathcal{T}_{P}\left(f_{0}, \cdots, f_{n}\right)=\mathcal{T}_{R}\left(e^{i q_{0}} f_{0}, \cdots, e^{i q_{n}} f_{n}\right)$. By induction on the degree, $\mathcal{T}_{R}$ satisfies the desired estimate, which is equivalent to the desired estimate for $\mathcal{T}_{P}$ since $\left\|e^{i q_{j}} f_{j}\right\|_{p_{j}}=\left\|f_{j}\right\|_{p_{j}}$.

If $P_{D}$ is nondegenerate, there exists a smallest integer $N$ such that $P_{D}\left(2^{N} x, 2^{N} y\right)$ has norm $\geq 1$ in the quotient space of homogeneous polynomials of degree $D$ modulo degenerate homogeneous polynomials of that degree, with respect to some fixed choice of norm on that finite-dimensional space. This norm is then also $\leq 2^{D}$.

By rescaling the variables $(x, y) \in \mathbb{R}^{\kappa+m}$ by a factor of $2^{N}$ we may reduce henceforth to the case $N=0$. Because $K$ is homogeneous of the critical degree and $\sum_{j=1}^{n} p_{j}^{-1}=q^{-1}$, such a rescaling does not affect the inequality to be proved.

By replacing $P_{D}$ by $P_{D}-\sum_{j} S_{j}\left(x+\ell_{j}(y)\right)$ for appropriate homogeneous real-valued polynomials $S_{j}$ of degree $D$, we may assume henceforth that $P_{D}$ has norm $\lesssim 1$ in the space of all homogeneous polynomials of degree $D$, rather than merely in the quotient space.

Fix a cutoff function $\zeta \in C_{0}^{\infty}\left(\mathbb{R}^{k}\right)$ which is $\equiv 1$ in some neighborhood of the origin, and consider the truncated multilinear functional $T_{P}^{\prime}\left(f_{1}, \cdots, f_{n}\right)(x)=\left\langle\zeta(\cdot) K e^{i P(x, \cdot)}, \prod_{j=1}^{n} f_{j}(x+\right.$ $\left.\left.\ell_{j}(\cdot)\right)\right\rangle$. Introduce also an auxiliary function $h \in C_{0}^{\infty}\left(\mathbb{R}^{d}\right)$ such that $\sum_{\nu \in \mathbb{Z}^{d}} h(x-\nu) \equiv 1$ and decompose

$$
T_{P}^{\prime}\left(f_{1}, \cdots, f_{n}\right)(x)=\sum_{\nu=\left(\nu_{0}, \cdots, \nu_{n}\right) \in \mathbb{Z}^{(n+1) d}}\left\langle\zeta(\cdot) K e^{i P(x, \cdot)}, \prod_{j=0}^{n} f_{j}\left(x+\ell_{j}(\cdot)\right) h\left(x+\ell_{j}(\cdot)-\nu_{j}\right)\right\rangle .
$$

Because of the presence of the compactly supported factor $\zeta(y)$, there exists $C_{0}<\infty$ such that any term with parameter $\nu$ vanishes unless $\max _{j}\left|\nu_{j}-\nu_{0}\right| \leq C_{0}$. Therefore by the triangle inequality (modified as above when $q<1$ ), Hölder's inequality, and the hypothesis 
$\sum_{j} p_{j}^{-1}=q^{-1}$, it suffices to prove the desired bound for fixed $\nu$, with $f_{j}(\cdot)$ replaced by $\tilde{f}_{j}(\cdot)=f_{j}(\cdot) h\left(\cdot-\nu_{j}\right)$, so long as a majorization uniform in $\nu$ is obtained. Henceforth we fix $\nu_{0}$, and assume that each function $f_{j}$ is supported within a fixed distance of $\nu_{0}$.

Make the change of variables $x=z+\nu_{0}$. Then $P(x, y)=P_{D}(x, y)+R(x, y)=P_{D}(z, y)+$ $\tilde{R}_{\nu_{0}}(z, y)$ where $\tilde{R}_{\nu_{0}}$ has degree $<D$. Moreover, if another cutoff function $\tilde{\zeta} \in C_{0}^{\infty}$ is chosen to be $\equiv 1$ in a sufficiently large neighborhood of the origin, then as a function of $z$,

$$
T_{P}^{\prime}\left(\tilde{f}_{1}, \cdots, \tilde{f}_{n}\right)(x)=\left\langle K(\cdot) e^{i \tilde{R}_{\nu_{0}}(z, \cdot)}\left(e^{i P_{D}(z, \cdot)} \zeta(\cdot) \tilde{\zeta}(z, \cdot)\right), \prod_{j=0}^{n} \tilde{f}_{j}\left(z+\ell_{j}(\cdot)\right)\right\rangle .
$$

Now the factor $\zeta(y) e^{i P_{D}(z, y)} \tilde{\zeta}(z, y)$ is smooth and compactly supported as a function of $(z, y)$, and is bounded above in any $C^{s}$ norm by a constant depending only on $s, D$ and the choice of $\tilde{\zeta}$. By induction on $D$,

$$
\left\|\left\langle K e^{i \tilde{R}_{\nu_{0}}(z, \cdot)}, \prod_{j=0}^{n} \tilde{f}_{j}\left(z+\ell_{j}(\cdot)\right)\right\rangle\right\|_{L^{q}(d z)} \leq C \prod_{j=1}^{n}\left\|\tilde{f}_{j}\right\|_{L^{p_{j}}},
$$

uniformly for all polynomials $\tilde{R}_{\nu_{0}}$ of degree $<D$.

Therefore we may invoke Lemma 6.2 to conclude that

$$
\left\|\left\langle K e^{i \tilde{R}_{\nu_{0}}(z, \cdot)}\left(e^{i P_{D}(z, \cdot)} \zeta(\cdot) \tilde{\zeta}(z, \cdot)\right), \prod_{j=0}^{n} \tilde{f}_{j}\left(z+\ell_{j}(\cdot)\right)\right\rangle\right\|_{L^{q}(d z)} \leq C \prod_{j}\left\|\tilde{f}_{j}\right\|_{L^{p_{j}}},
$$

uniformly in $\nu$.

It remains to analyze

$$
\left\langle K e^{i P}(1-\zeta(\cdot)), \prod_{j} f_{j}\left(x+\ell_{j}(\cdot)\right)\right\rangle=\sum_{r=0}^{\infty}\left\langle K e^{i P} \eta\left(2^{-r} \cdot\right), \prod_{j} f_{j}\left(x+\ell_{j}(\cdot)\right)\right\rangle
$$

where $\eta(y)=\zeta(y / 2)-\zeta(y)$. We claim that

$$
\left\|\left\langle K e^{i P} \eta\left(2^{-r} \cdot\right), \prod_{j} f_{j}\left(x+\ell_{j}(\cdot)\right)\right\rangle\right\|_{q} \leq C 2^{-\varepsilon r} \prod_{j}\|f\|_{p_{j}}
$$

for some $\varepsilon>0$ and $C<\infty$ depending only on $D,\left\{V_{j}\right\}$. Once (6.5) is proved, the proof may immediately be completed if $q \geq 1$, by invoking the triangle inequality. When $0<$ $q<1$, the rapidly decaying factor $C 2^{-\varepsilon r}$, together with the modified triangle inequality $\left|\sum_{i} b_{i}\right|^{q} \leq \sum_{i}\left|b_{i}\right|^{q}$, likewise suffice to complete the proof.

Because of the homogeneity of $K$ and the condition $\sum_{j} p_{j}^{-1}=q^{-1},(6.5)$ is equivalent to

$$
\left\|\left\langle K(\cdot) \eta(\cdot) e^{i P_{r}}, \prod_{j} f_{j}\left(x+\ell_{j}(\cdot)\right)\right\rangle\right\|_{L^{q}(d x)} \leq C 2^{-\varepsilon r} \prod_{j}\|f\|_{L^{p_{j}}}
$$

via the substitution $(x, y)=\left(2^{r} x^{\prime}, 2^{r} y^{\prime}\right)$, where $P_{r}(x, y)=P\left(2^{r} x, 2^{r} y\right)=2^{D r} P_{D}(x, y)+$ $\tilde{R}_{r}(x, y)$, and the remainder term $\tilde{R}_{r}$ has degree strictly less than $D$ and is real-valued.

As above, by introducing a partition of unity we may reduce matters to the case where all $f_{j}$ are supported in a fixed bounded set, by introducing a partition of unity. $\tilde{R}_{r}$ is thereby further modified and depends also on the index $\nu_{0}$; it satisfies no useful upper or lower bounds but still has degree strictly $<D$.

Any such polynomial $2^{D r} P_{D}+\tilde{R}$, where $\tilde{R}$ has degree $<D$, has norm $\geq c 2^{D r}$ in the quotient space of polynomials of degree $\leq D$ modulo degenerate polynomials of degreee $\leq D$, for some fixed constant $c>0$, depending only on $D$. The function $K \eta$ is Lipschitz, 
since $K$ is a Calderón-Zygmund kernel and $\eta$ vanishes identically in some neighborhood of the origin. Therefore (6.6) follows from the assumption that $\left\{V_{j}\right\}$ has the uniform decay property.

\section{FURTHER RESUlTS}

Theorem 2.4 is a direct consequence of Lemma 3.5 together with Theorem 2.3. The next result extends Theorem 2.2 .

Theorem 7.1. Let $\left\{V_{j}\right\}$ be any finite collection of subspaces of $\mathbb{R}^{m}$, and let $\left\{W_{i}\right\}$ be any finite collection of codimension one subspaces of $\mathbb{R}^{m}$. If $\left\{V_{j}\right\}$ has the uniform decay property, then so does $\left\{V_{j}\right\} \cup\left\{W_{i}\right\}$.

Proof. It suffices to prove this in the case where a single codimension one subspace $W$ is given. Choose coordinates with respect to which $W=\left\{\left(x^{\prime}, x_{m}\right): x_{m}=0\right\}$. Let $P$ be any nondegenerate polynomial, relative to the augmented collection $\left\{V_{j}\right\} \cup\{W\}$. It is no loss of generality to assume that no subspaces $V_{j}$ are contained in $W$, for any such spaces may be deleted from $\left\{V_{j}\right\}$ without affecting the nondegeneracy of $P$.

$\partial P / \partial x_{m}$ is nondegenerate relative to $\left\{V_{j}\right\}$. For if not, then there exists a polynomial decomposition $\partial P / \partial x_{m}=\sum_{j} q_{j} \circ \pi_{j}$. Since no space $V_{j}$ is contained in $W$, there exist nonzero vectors $v_{j} \in V_{j}$ such that $\partial\left(f \circ \pi_{j}\right) / \partial x_{m}=\left(v_{j} \cdot \nabla f\right) \circ \pi_{j}$ for all functions $f: V_{j} \rightarrow \mathbb{R}$. Since $0 \neq v_{j} \in V_{j}$, there exist polynomials $Q_{j}$ such that $v_{j} \cdot \nabla Q_{j} \equiv q_{j}$. Consequently $\partial\left(Q_{j} \circ \pi_{j}\right) / \partial x_{m}=q_{j} \circ \pi_{j}$, and hence $\tilde{P}=\sum_{j} Q_{j} \circ \pi_{j}$ satisfies $\partial(P-\tilde{P}) / \partial x_{m} \equiv 0$. Thus $P-\sum_{j} Q_{j} \circ \pi_{j}$ is a function of $x_{m}$ alone, contradicting the hypothesized nondegeneracy of $P$.

It follows that there exists $z \in \mathbb{R}^{m}$ for which $P_{z}(x)=P\left(x^{\prime}, x_{m}\right)-P\left(x^{\prime}, x_{m}+z\right)$ is nondegenerate relative to $\left\{V_{j}\right\}$. For if not, since $\partial P / \partial x_{m}$ can be reconstructed from $\left\{P_{z_{i}}\right\}$ for a suitable finite collection of points $z_{i}$ by the Lagrange interpolation formula, degeneracy of each $P_{z_{i}}$ implies degeneracy of $\partial P / \partial x_{m}$.

Consider the quotient space $\mathcal{P}$ of all polynomials of degrees not exceeding the degree of $P$ modulo the subspace of all such polynomials which are degenerate relative to $\left\{V_{j}\right\}$, and equip it some inner product structure. Then $\left\|P_{z}\right\|_{\mathcal{P}}^{2}$ is a polynomial in $z$ which does not vanish identically. Hence there exist $C, \delta \in \mathbb{R}^{+}$such that for any ball $B$ of fixed finite radius, for any $\varepsilon>0$,

$$
\left|\left\{z \in B:\left\|P_{z}\right\|_{\mathcal{P}}^{2}<\varepsilon\right\}\right| \leq C \varepsilon^{\delta} .
$$

We may now argue as in the proof of Theorem 2.1 to conclude the proof that $P$ has the decay property.

To establish the uniform decay property, fix $c \in(1, \infty$ and a degree $d$. Let $P$ be any polynomial of degree $\leq d$ whose norm, in the quotient space of polynomials of degrees $\leq d$ modulo polynomials of degrees $\leq d$ that are degenerate relative to $\left\{V_{j}\right\} \cup\{W\}$, lies in $\left[c^{-1}, c\right]$. Since this quotient space is a finite-dimensional vector space, $P$ thus belongs to a compact subset. Together with the above reasoning, this implies that (7.1) holds with a constant depending on $d, c$ but not on $P$. The proof of Theorem 2.1 then yields a decay estimate independent of $P$.

Corollary 7.2. Let a real-valued polynomial $P$ be nondegenerate relative to a finite collection $\left\{V_{j}\right\}$ of subspaces of $\mathbb{R}^{m}$, and suppose that $V_{j}$ has codimension one for all $j>1$. Then 
$\left\{V_{j}\right\}$ has the uniform decay property. Moreover

$$
\left|\Lambda_{\lambda}\left(f_{1}, \cdots, f_{n}\right)\right| \leq C|\lambda|^{-\varepsilon}\left\|f_{1}\right\|_{2} \prod_{j>1}\left\|f_{j}\right\|_{\infty},
$$

with uniform bounds if $P$ belongs to a family of uniformly nondegenerate polynomials.

A decay estimate with $\prod_{j}\left\|f_{j}\right\|_{\infty}$ on the right-hand side of the inequality follows by repeated applications of the preceding theorem. (7.2) then follows by interpolation with the trivial bound $\left|\Lambda_{\lambda}\left(f_{1}, \cdots, f_{n}\right)\right| \lesssim\left\|f_{1}\right\|_{1} \prod_{j>1}\left\|f_{j}\right\|_{\infty}$.

With Corollary 7.2 in hand, the proof of Theorem 2.1 can be reorganized in a simpler way, though without altering the main idea, as follows. We are given a collection of onedimensional subspaces $\left\{V_{j}: 1 \leq j \leq n\right\}$ of $\mathbb{R}^{m}$, lying in general position, and it is assumed that $n<2 m$. Again we discuss only the most extreme case where $n=2 m-1$. Let $P$ be some polynomial which is nondegenerate relative to $\left\{V_{j}\right\}$.

We will prove power decay in the form (7.2). Let $W$ be the span of those $V_{j}$ with $2 \leq j \leq m$, and likewise let $\tilde{W}$ be the span of those with $m<j \leq n=2 m-1$. By the general position hypothesis, $W$ and $\tilde{W}$ are distinct subspaces of codimension one, neither of which contains $V_{1}$.

Let functions $f_{1} \in L^{2}\left(V_{1}\right)$, and $f_{j} \in L^{\infty}\left(V_{j}\right)$ for $j>1$, be given. As usual, we may assume each $f_{j}$ to be supported in a fixed bounded subset of $V_{j}$.

The proof is divided into two main cases, depending on whether or not $f_{1}$ is $\lambda$-uniform, as defined in Definition 5.1; the parameter $d$ appearing in that definition is here taken to be the degree of $P$. The case where $f_{1}$ is not $\lambda$-uniform is handled as already discussed in the proof of Theorem 2.1.

Let $A(\lambda)$ be the best constant in the inequality (7.2). Define functions $F, \tilde{F}$ on $W, \tilde{W}$ respectively by the relations $F \circ \pi=\prod_{j=2}^{m} f_{j} \circ \pi_{j}, \tilde{F} \circ \tilde{\pi}=\prod_{j>m} f_{j} \circ \pi_{j}$ where $\pi, \tilde{\pi}$ denote the orthogonal projections from $\mathbb{R}^{m}$ onto $W, \tilde{W}$ respectively. Then $\|F\|_{2} \lesssim \prod_{j=2}^{m}\left\|f_{j}\right\|_{\infty}$ and $\|\tilde{F}\|_{2} \lesssim \prod_{j>m}\left\|f_{j}\right\|_{\infty}$. Our multilinear oscillatory integral is

$$
\Lambda_{\lambda}\left(f_{1}, \cdots, f_{n}\right)=\int_{\mathbb{R}^{m}} e^{i \lambda P(x)}(F \circ \pi)(\tilde{F} \circ \tilde{\pi})\left(f_{1} \circ \pi_{1}\right) \eta d x .
$$

If $P$ happens to be nondegenerate relative to the collection of three subspaces $\left\{V_{1}, W, \tilde{W}\right\}$, then the proof is complete by virtue of Corollary 7.2, in its more precise version (7.2). If not, then $P=p \circ \pi+\tilde{p} \circ \tilde{\pi}+q \circ \pi_{1}$ for certain polynomials $p, \tilde{p}, q$, whose degrees do not exceed the degree of $P$.

Suppose without loss of generality that $\left\|f_{1}\right\|_{L^{2}}=1$, and that $f_{1}$ is $\lambda$-uniform. Set $G=e^{i \lambda p} F, H=e^{i \lambda \tilde{p}} \tilde{F}$, and $g=e^{i \lambda q} f_{1}$. Our integral becomes

$$
\Lambda_{\lambda}=\int(G \circ \pi)(H \circ \tilde{\pi})\left(g \circ \pi_{1}\right) \eta=c \iiint \widehat{G}(\xi) \widehat{H}(\tilde{\xi}) \widehat{g}(\zeta) \widehat{\eta}\left(\pi^{*} \xi+\tilde{\pi}^{*} \tilde{\xi}+\pi_{1}^{*} \zeta\right) d \xi d \tilde{\xi} d \zeta
$$

where $\pi_{1}^{*}$ is the adjoint of $\pi_{1}: \mathbb{R}^{m} \rightarrow V_{1}$, and analogously for $\pi^{*}, \tilde{\pi}^{*}$; here $\zeta \in \mathbb{R}^{1}, \xi \in \mathbb{R}^{m}$, and $\tilde{\xi} \in \mathbb{R}^{m}$. The $\lambda$-uniformity condition gives a bound $\|\widehat{g}\|_{L^{\infty}}=O\left(|\lambda|^{-\tau}\right)$ for some fixed $\tau>0$. Therefore for $|\lambda| \geq 1$

$$
\left|\Lambda_{\lambda}\right| \lesssim|\lambda|^{-\tau} \iiint|\widehat{G}(\xi) \widehat{H}(\tilde{\xi})| \cdot\left|\widehat{\eta}\left(\pi^{*} \xi+\tilde{\pi}^{*} \tilde{\xi}+\pi_{1}^{*} \zeta\right)\right| d \xi d \tilde{\xi} d \zeta
$$


It therefore suffices to have

$$
\sup _{\tilde{\xi}} \iint\left|\widehat{\eta}\left(\pi^{*} \xi+\tilde{\pi}^{*} \tilde{\xi}+\pi_{1}^{*} \zeta\right)\right| d \xi d \zeta<\infty
$$

and the same with the roles of $\tilde{\xi}, \xi$ interchanged. (7.3) holds because $\eta$ may be taken to belong to $C_{0}^{m+1}$, so that $\widehat{\eta}$ decays rapidly, and the mapping $\mathbb{R} \times \mathbb{R}^{m-1} \ni(\zeta, \xi) \rightarrow \pi^{*} \xi+\pi_{1}^{*} \zeta \in$ $\mathbb{R}^{m}$ is linear and injective.

\section{REFERENCES}

[1] A. Carbery, M. Christ, and J. Wright, Multidimensional van der Corput and sublevel set estimates, J. Amer. Math. Soc. 12 (1999), no. 4, 981-1015.

[2] W. T. Gowers, A new proof of Szemerédi's theorem for arithmetic progressions of length four, Geom. Funct. Anal. 8 (1998), no. 3, 529-551.

[3] _ Arithmetic progressions in sparse sets, Current developments in mathematics, 2000, 149-196, Int. Press, Somerville, MA, 2001.

[4] M. Lacey and C. Thiele, $L^{p}$ estimate on the bilinear Hilbert transform, Ann. of Math. 146 (1997), 693-724.

[5] — On Calderón's conjecture, Ann. of Math. 149 (1999), 475-496.

[6] C. Muscalu, T. Tao, and C. Thiele, Multi-linear operators given by singular multipliers, J. Amer. Math. Soc. 15 (2002), 469-496.

[7] D. H. Phong and E. M. Stein, Operator versions of the van der Corput lemma and Fourier integral operators, Math. Res. Lett. 1 (1994), 27-33.

[8] D. H. Phong, E. M. Stein, and J. Sturm, Multilinear level set operators, oscillatory integral operators, and Newton polyhedra, Math. Ann. 319 (2001), no. 3, 573-596.

[9] F. Ricci and E. M. Stein, Harmonic analysis on nilpotent groups and singular integrals, J. Funct. Anal. 73 (1987), 179-194.

[10] E. M. Stein, Harmonic analysis: real-variable methods, orthogonality, and oscillatory integrals. With the assistance of Timothy S. Murphy, Princeton Mathematical Series, 43. Monographs in Harmonic Analysis, III. Princeton University Press, Princeton, NJ, 1993.

Michael Christ, Department of Mathematics, University of California, Berkeley, CA 94720-3840, USA

E-mail address: mchrist@math.berkeley.edu

Xiaochun Li, School of Mathematics, IAS, Princeton, NJ, 08540

E-mail address: xcli@ias.edu

Terence Tao, Department of Mathematics, UClA, Los Angeles, CA 90095-1555, USA

E-mail address: tao@math.ucla.edu

Christoph Thiele, Department of Mathematics, UCla, Los Angeles, CA 90095-1555, USA

E-mail address: thiele@math.ucla.edu 